\title{
Cuntz-Krieger Algebras and Wavelets on Fractals
}

\author{
Matilde Marcolli • Anna Maria Paolucci
}

Received: 6 August 2009 / Accepted: 2 November 2009 / Published online: 21 November 2009 (C) The Author(s) 2009. This article is published with open access at Springerlink.com

\begin{abstract}
We consider representations of Cuntz-Krieger algebras on the Hilbert space of square integrable functions on the limit set, identified with a Cantor set in the unit interval. We use these representations and the associated Perron-Frobenius and Ruelle operators to construct families of wavelets on these Cantor sets.
\end{abstract}

Keywords Cuntz-Krieger · Wavelets · Fractals · Cantor set · Hilbert space · Perron-Frobenius operator $\cdot$ Ruelle operator

\section{Contents}

1 Introduction . . . . . . . . . . . . . . . . . . . . . . . . 42

2 Representations of Cuntz-Krieger Algebras . . . . . . . . . . . . . . . . . . . . . . . . . . . . . . . 43

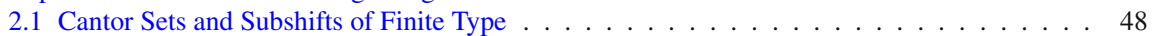

2.2 Perron-Frobenius Operator . . . . . . . . . . . . . . . . . . . . . . . . . . . . 49

2.3 Projection Valued Measures . . . . . . . . . . . . . . . . . . . . . . . . . . . 54

2.4 Hausdorff Dimension . . . . . . . . . . . . . . . . . . . . . . . . . . . . 57

2.5 Real Valued Measures and Fourier Transforms . . . . . . . . . . . . . . . . . . . . . . . 61

2.6 Sierpinski Fractals . . . . . . . . . . . . . . . . . . . . . . . . . . . . 64

3 Wavelets on Fractals . . . . . . . . . . . . . . . . . . . . . . . . 68

4 Ruelle Transfer Operator . . . . . . . . . . . . . . . . . . . . . . . . . . . . . . . . . . 72

4.1 Random Processes . . . . . . . . . . . . . . . . . . . . . . . . 73

4.2 A Trigonometric Example . . . . . . . . . . . . . . . . . . . . . . 75

Communicated by Palle Jorgensen.

M. Marcolli

Department of Mathematics, California Institute of Technology, Pasadena, CA 91125, USA e-mail:matilde@caltech.edu

A. M. Paolucci $(\varangle)$

Max Planck Institute for Mathematics, Vivatsgasse 7, 53111 Bonn, Germany

e-mail: paolucci@mpim-bonn.mpg.de 
5 Examples and Applications _. . . . . . . . . . . . . . . . . . . . . . . . 75

5.1 Hensley Cantor Sets and Continued Fraction Expansion . . . . . . . . . . . . . . . . . 75

5.2 Graph Wavelets from Cuntz-Krieger Algebras . . . . . . . . . . . . . . . . . . . . . . . 77

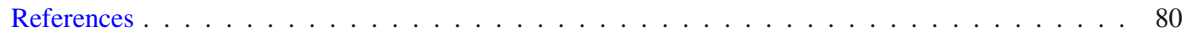

\section{Introduction}

A class of representations of the Cuntz algebra $O_{N}$ called permutative representations were studied and classified in $[3,4,10]$. Besides interest in their own right within the field of operator algebras, Cuntz algebras representations have very interesting applications to wavelets, fractals, and dynamical systems, see [3,4]. Some of these results have been extended to the more general class of Cuntz-Krieger algebras (see [20-22]), where representations of these algebras are related to Perron-Frobenius operators of certain measure space transformations. Similar representations of Cuntz-Krieger algebras were considered in the context of limit sets of Schottky groups and actions on trees in [5-7] for arithmetic applications to Arakelov geometry and p-adic Mumford curves.

In this paper we look at representations of the Cuntz-Krieger algebra having a underlying self-similarity structure. The concept of self-similarity has proved to be fundamental in mathematics as well as in diverse applications, related to the renormalization of structures on nested families of scales. In the theory of wavelets, the scales may be represented in resolutions taking the form of nested systems of linear spaces, while in $C^{*}$-algebra theory it gives rise to representations of algebras on generators and relations such as those that define the Cuntz and Cuntz-Krieger algebras.

Cuntz-Krieger algebras arise naturally from semibranching function systems on measure spaces, where the partial inverses $\sigma_{i}$ of the coding map $\sigma$ are not defined everywhere. The resulting algebra is generated by partial isometries $S_{i}$ associated to the maps in the semibranching function system, and the relations between these generators involve a matrix $A$ with entries equal to zero or one, which describes the decomposition of the domains of the $\sigma_{i}$ as a union of ranges of other $\sigma_{j}$ in the same family.

Conversely, a Cuntz-Krieger algebra $O_{A}$ defined by generators and relations in terms of an $N \times N$-matrix $A$ as above determines a semibranching function system on the limit set of infinite sequences in an alphabet on $N$ letters with the admissibility condition that consecutive letters $i j$ can appear in a word if and only if the corresponding entry in the matrix is $A_{i j}=1$. One can identify this limit set $\Lambda_{A}$ as a Cantor set inside the interval $[0,1]$ by considering points whose $N$-adic digital expansion satisfies the admissibility condition. On this Cantor set the action of the maps $\sigma_{i}$ become simple shifts in the $N$-adic expansion and the representation of $O_{A}$ on the Hilbert space $L^{2}\left(\Lambda_{A}, \mu\right)$, with respect to the Hausdorff measure of the appropriate dimension, has an especially simple form, and so does also the Perron-Frobenius operator for the shift map $\sigma$, which is expressed in terms of the generators of the algebra.

The Hausdorff dimension of the limit set $\Lambda_{A}$ is computed using the PerronFrobenius theorem for the non-negative matrix $A$, which also shows that the components of the Perron-Frobenius eigenvector of $A$ give the measures of the ranges of the maps $\sigma_{i}$ in the normalized Hausdorff measure of dimension the Hausdorff dimension of $\Lambda_{A}$, which is the unique probability measure satisfying the self-similarity condition for the fractal set $\Lambda_{A}$. 
The Perron-Frobenius eigenvector of the matrix $A^{t}$ determines a fixed point for the Perron-Frobenius operator for the shift map $\sigma$ on the limit set $\Lambda_{A}$, which in turn gives a KMS state for an associated time evolution on the algebra $O_{A}$ at inverse temperature equal to the Hausdorff dimension of $\Lambda_{A}$.

One can construct as in [17] further measures on $\Lambda_{A}$, using operator valued measures and square-integrable functions of unit norm. As in the case of the Cuntz algebras, by analyzing the Fourier transforms of these measures, one sees that one can approximate them with Dirac measures supported at truncations of the $N$-adic expansions.

Besides the Cantor set $\Lambda_{A} \subset[0,1]$, there is another fractal set that one can associate to the same matrix $A$, namely a Sierpinski fractal $\mathbb{S}_{A}$ inside the unit cube, given by points $(x, y)$ whose digits in the $N$-adic expansion satisfy the condition that $A_{x_{i} y_{i}}=1$. The Hausdorff dimension of these sets is simply computed in terms of the number of non-zero entries in $A$. The shifts in the $N$-adic expansion determine a semibranching function systems on $\mathbb{S}_{A}$, where, unlike in the case of $\Lambda_{A}$, the maps are everywhere defined, hence they give rise to an action of a Cuntz algebra or rank depending on the number of non-zero entries in $A$. There is a natural embedding of $\Lambda_{A}$ into $\mathbb{S}_{A}$ induced by the shift map on $\Lambda_{A}$. The action of the Cuntz algebra determines via this embedding of $\Lambda_{A}$ into $\mathbb{S}_{A}$ an action of a Cuntz-Krieger algebra.

We show how to use the representation of the algebra $O_{A}$ to construct an orthonormal system of wavelets on $L^{2}\left(\Lambda_{A}, d \mu\right)$.

We then consider the Ruelle transfer operator for the shift $\sigma$ on $\Lambda_{A}$, with nonnegative valued potential $W$ satisfying the Keane condition that the sum of the values over preimages under $\sigma$ adds up to one. We show that one can construct from these measures on $\Lambda_{A^{t}}$, for the transpose matrix $A^{t}$, in terms of random walks where the probabilities assigned to words of a given length in the alphabet depends upon the values of the potential $W$. A simple example of a potential satisfying the Keane condition is given in terms of trigonometric functions.

The example of the continued fraction expansion on the Hensley Cantor sets considered in $[24,25]$ is described as an example where the general results of this paper can be applied.

As an application we also show how the technique we described to construct wavelets on the Cantor sets $\Lambda_{A}$ can be adapted to construct families of graph wavelets, using Cuntz-Krieger algebras associated to finite graphs with no sinks. Graph wavelets are considered a useful tool for spatial network traffic analysis [8].

\section{Representations of Cuntz-Krieger Algebras}

Let $A$ be an $N \times N$ matrix with entries in $\{0,1\}$. For consistency with the notation we adopt later in the paper, it is convenient to index the entries $A=\left(A_{i j}\right)$ with indices $i, j \in\{0, \ldots, N-1\}$ instead of $\{1, \ldots, N\}$.

Recall that the Cuntz-Krieger algebra $O_{A}$ associated to such a matrix $A$ is the $C^{*}$-algebra generated by $N$ (non-zero) partial isometries $S_{0}, \ldots, S_{N-1}$ satisfying the relations

$$
S_{i}^{*} S_{i}=\sum_{j} A_{i j} S_{j} S_{j}^{*}
$$


and

$$
\sum_{i=0}^{N-1} S_{i} S_{i}^{*}=1 .
$$

The algebra $O_{A}$ is uniquely determined by the relations (2.1) and (2.2) and it is linearly spanned by the set of $S_{\alpha} S_{\beta}^{\star}$ with words $\alpha$ and $\beta$ in $\{0, \ldots, N-1\}$ with possibly different lengths $|\alpha|$ and $|\beta|$, see [9].

We are especially interested here in representations of $O_{A}$ as bounded operators on Hilbert spaces of the form $\mathcal{H}=L^{2}(X, \mu)$, for $(X, \mu)$ a measure space. The representations we are interested in, which include the cases of the arithmetic applications mentioned above, are all constructed in terms of what we refer to as a semibranching function system, which will be concretely realized in terms of a shift map on a Cantor-like fractal set and its partial inverses.

Definition 2.1 Consider a measure space $(X, \mu)$ and a finite family $\left\{\sigma_{i}\right\}_{i \in I}$, \#I=N, of measurable maps $\sigma_{i}: D_{i} \rightarrow X$, defined on measurable subsets $D_{i} \subset X$. The family $\left\{\sigma_{i}\right\}$ is a semibranching function system if the following holds.

(1) There exists a corresponding family $\left\{D_{i}\right\}_{i=1}^{N}$ of measurable subsets of $X$ with the property that

$$
\mu\left(X \backslash \cup_{i} R_{i}\right)=0, \quad \text { and } \quad \mu\left(R_{i} \cap R_{j}\right)=0, \quad \text { for } \quad i \neq j,
$$

where we denote by $R_{i}$ the range $R_{i}=\sigma_{i}\left(D_{i}\right)$.

(2) There is a Radon-Nikodym derivative

$$
\Phi_{\sigma_{i}}=\frac{d\left(\mu \circ \sigma_{i}\right)}{d \mu}
$$

with $\Phi_{\sigma_{i}}>0, \mu$-almost everywhere on $D_{i}$.

A measurable map $\sigma: X \rightarrow X$ is called a coding map for the family $\left\{\sigma_{i}\right\}$ if $\sigma \circ \sigma_{i}(x)=$ $x$ for all $x \in D_{i}$.

Thus, the maps of the semibranching function system are partial inverses of the coding map $\sigma$. Notice that the reverse composition $\sigma_{i} \circ \sigma$ is only defined when the image of $x$ under $\sigma$ lands in the domain $D_{i}$ of $\sigma_{i}$.

Given a semibranching function system $\left\{\sigma_{i}\right\}_{i=0}^{N-1}$ with coding map $\sigma$, one can construct an associated family of linear operators $\left\{T_{i}\right\}_{i=0}^{N-1}$ acting on the Hilbert space $L^{2}(X, \mu)$ by setting

$$
\left(T_{i} \psi\right)(x)=\chi_{R_{i}}(x)\left(\Phi_{\sigma_{i}}(\sigma(x))\right)^{-1 / 2} \psi(\sigma(x))
$$

with $\psi \in L^{2}(X, \mu)$, where $\chi_{R_{i}}$ is the characteristic function of $R_{i} \subset X$. 
Lemma 2.2 The adjoint of the operator $T_{i}$ of (2.4) is of the form

$$
\left(T_{i}^{\star} \xi\right)(x)=\chi D_{i}(x)\left(\Phi_{\sigma_{i}}(x)\right)^{1 / 2} \xi\left(\sigma_{i}(x)\right) .
$$

Proof We have

$$
\begin{aligned}
\left\langle T_{i} \psi, \xi\right\rangle & =\int_{R_{i}} \overline{\left(\Phi_{\sigma_{i}}(\sigma(x))\right)^{-1 / 2} \psi(\sigma(x))} \xi(x) d \mu(x) \\
& =\int_{D_{i}} \overline{\left(\Phi_{\sigma_{i}}(u)\right)^{-1 / 2} \psi(u)} \xi\left(\sigma_{i}(u)\right) \frac{d \mu \circ \sigma_{i}}{d \mu} d \mu(u) \\
& =\int_{D_{i}} \overline{\psi(u)}\left(\Phi_{\sigma_{i}}(u)\right)^{1 / 2} \xi\left(\sigma_{i}(u)\right) d \mu(x)=\left\langle\psi, T_{i}^{*} \xi\right\rangle,
\end{aligned}
$$

where we used the fact that the $\Phi_{\sigma_{i}}$ are positive real valued. This gives (2.5).

We then see easily that the operators $T_{i}$ and $T_{i}^{*}$ satisfy the following relation.

Proposition 2.3 The operators $T_{i}$ of (2.4) and their adjoints (2.5) satisfy the relations $T_{i} T_{i}^{*}=P_{i}$, where $P_{i}$ is the projection given by multiplication by $\chi_{R_{i}}$. This gives $\sum_{i} T_{i} T_{i}^{*}=1$. Similarly, $T_{i}^{*} T_{i}=Q_{i}$, where $Q_{i}$ is the projection given by multiplication by the characteristic function $\chi_{D_{i}}$.

Proof We write explicitly the action of the operator $T_{i} T_{i}^{*}$ on elements $\xi \in L^{2}(X, d \mu)$. We have

$$
\left(T_{i} T_{i}^{*} \xi\right)(x)=\chi_{R_{i}}(x) \chi_{D_{i}}(\sigma(x)) \Phi_{\sigma_{i}}^{-1 / 2}(\sigma(x)) \Phi_{\sigma_{i}}^{1 / 2}(\sigma(x)) \xi(x)=\chi_{R_{i}}(x) \xi(x) .
$$

Equivalently, we can write

$$
\begin{aligned}
\left\langle T_{i}^{*} \xi, T_{i}^{*} \xi\right\rangle & =\int_{D_{i}} \Phi_{\sigma_{i}}(x)\left|\xi\left(\sigma_{i}(x)\right)\right|^{2} d \mu(x) \\
& =\int_{R_{i}} \Phi_{\sigma_{i}}(\sigma(u))|\xi(u)|^{2} \frac{d \mu \circ \sigma}{d \mu} d \mu(u) .
\end{aligned}
$$

Notice then that one has

$$
\left.\frac{d \mu \circ \sigma}{d \mu}\right|_{R_{i}}=\left(\Phi_{\sigma_{i}} \circ \sigma\right)^{-1}
$$

so that we obtain

$$
\left\langle T_{i}^{*} \xi, T_{i}^{*} \xi\right\rangle=\int_{R_{i}}|\xi(u)|^{2} d \mu(u)=\left\langle P_{i} \xi, P_{i} \xi\right\rangle
$$


which gives $T_{i} T_{i}^{*}=P_{i}$, the range projection on $L^{2}(X, \mu)$ realized by the multiplication operator by the characteristic function of the set $R_{i}$. By the assumptions (2.3) on the semibranching function system we know that the projections $P_{i}$ are orthogonal and that $\sum_{i} P_{i}=1$.

We then consider the product $T_{i}^{*} T_{i}$.

We have

$$
\left\langle T_{i} \xi, T_{i} \xi\right\rangle=\int_{R_{i}} \Phi_{\sigma_{i}}^{-1}(\sigma(x))|\xi(\sigma(x))|^{2} d \mu(x)
$$

If $x \in R_{i}$ then $\sigma(x) \in D_{i}$ since $\sigma \circ \sigma_{i}=i d$ on $D_{i}$. Thus, we write the above as

$$
\int_{D_{i}} \Phi_{\sigma_{i}}^{-1}(u)|\xi(u)|^{2}\left(\frac{d \mu \circ \sigma}{d \mu}\right)^{-1} d \mu(u)=\int_{D_{i}}|\xi(u)|^{2} d \mu(u)=\left\langle\chi_{D_{i}} \xi, \chi_{D_{i}} \xi\right\rangle
$$

where we used again (2.6). This gives $T_{i}^{*} T_{i}=Q_{i}$, where $Q_{i}$ is the domain projection given by multiplication by the characteristic function $\chi_{D_{i}}$. Unlike the range projections $P_{i}$, the domain projections $Q_{i}$ are, in general, not orthogonal.

When the maps $\sigma_{i}$ are defined everywhere on $X$, one obtains from the operators $T_{i}$ and $T_{i}^{*}$ a representation of the Cuntz algebra $O_{N}$ in the following way.

Proposition 2.4 Let $\left\{\sigma_{i}\right\}$ be a semibranching function system on $X$, where the $\sigma_{i}$ are defined on all of $X$, that is, $D_{i}=X$ for all $i=0, \ldots, N-1$. Then the operators $T_{i}$ define a representation of the Cuntz algebra $O_{N}$ on the Hilbert space $\mathcal{H}=L^{2}(X, \mu)$. Namely, they satisfy the relations

$$
T_{i}^{*} T_{i}=1, \quad \sum_{i} T_{i} T_{i}^{*}=1
$$

Proof Under the assumption that the semibranching function system has $D_{i}=X$ for all $i \in I$, we obtain from Proposition 2.3 above that the operators $T_{i}$ and $T_{i}^{*}$ of (2.4) and (2.5) satisfy $T_{i}^{*} T_{i}=1$. Moreover, we know from Proposition 2.3 that $T_{i} T_{i}^{*}=P_{i}$, the range projections given by multiplication by the characteristic functions $\chi_{R_{i}}$. Since these range projections are orthogonal and the union of the $R_{i}$ exhausts $X$ up to sets of measure zero, we obtain that $\sum_{i} T_{i} T_{i}^{*}=1$.

In the case where the maps $\sigma_{i}$ are not defined everywhere on $X$, but only on smaller domains $D_{i} \subset X$, one can then use the operators $T_{i}$ and $T_{i}^{*}$ of (2.4) and (2.5) to construct representations of Cuntz-Krieger algebras, when the domains $D_{i}$ have the property that

$$
\chi_{D_{i}}=\sum_{j} A_{i j} \chi_{R_{j}}
$$

The examples considered in [5-7] are particular cases of this general procedure. 
Proposition 2.5 Let $\left\{\sigma_{i}\right\}$ be a semibranching function system on $X$, where the $\sigma_{i}$ are defined on subsets $D_{i} \subset X$ satisfying (2.8) (possibly up to sets of measure zero). Also assume that $A_{i i}=1$ for all $i=0, \ldots, N-1$. Then the operators $T_{i}$ and $T_{i}^{*}$ of (2.4) and (2.5) satisfy the Cuntz-Krieger relations (2.1) and (2.2), namely

$$
\sum_{i} T_{i} T_{i}^{*}=1 \quad \text { and } \quad T_{i}^{*} T_{i}=\sum_{j} A_{i j} T_{i} T_{i}^{*}
$$

hence they determine a representation of the Cuntz-Krieger algebra $O_{A}$ on the Hilbert space $\mathcal{H}=L^{2}(X, \mu)$.

Proof Using (2.5) and (2.8) we have

$$
\left(T_{i}^{*} \xi\right)(x)=\sum_{j} A_{i j} \chi_{R_{j}}(x) \Phi_{\sigma_{i}}^{1 / 2}(x) \xi\left(\sigma_{i}(x)\right) .
$$

We then obtain

$$
\begin{aligned}
\left(T_{i} T_{i}^{*} \xi\right)(x) & =\sum_{j} A_{i j} \chi_{R_{i}}(x) \chi_{R_{j}}(\sigma(x)) \Phi_{\sigma_{i}}^{-1 / 2}(\sigma(x)) \Phi_{\sigma_{i}}^{1 / 2}(\sigma(x)) \xi(x) \\
& =\sum_{j} A_{i j} \chi_{R_{i j}}(x) \xi(x)=P_{i} \xi(x),
\end{aligned}
$$

since we have from (2.8) that

$$
\cup_{j: A_{i j}=1} R_{i j}=\left\{x \in R_{i} \mid \sigma(x) \in D_{i}\right\}=R_{i} .
$$

Since the projections $P_{i}$ are orthogonal, we then obtain

$$
\sum_{i} T_{i} T_{i}^{*}=1
$$

This gives (2.2) with $S_{i}=T_{i}$. Similarly, we have

$$
T_{i}^{*} T_{i}=Q_{i}
$$

from Proposition 2.3, where $Q_{i}$ is the projection given by multiplication by $\chi_{D_{i}}$. Using again (2.8) this then gives

$$
T_{i}^{*} T_{i}=\sum_{j} A_{i j} P_{j}=\sum_{j} T_{i} T_{i}^{*}
$$

which gives (2.1) with $S_{i}=T_{i}$.

We describe below an important special case of semibranching function system, which gives rise to representations of Cuntz-Krieger algebras of the type described in Proposition 2.5. 


\subsection{Cantor Sets and Subshifts of Finite Type}

Let $\mathfrak{A}$ be an alphabet in $N$ letters, which we can identify with the set $\{0, \ldots, N-1\}$. Let $\Lambda_{A}$ be the set of all infinite admissible words in the alphabet $\mathfrak{A}$, where the admissibility condition is specified by an $N \times N$ matrix $A$ with entries in $\{0,1\}$. Namely,

$$
\Lambda_{A}:=\left\{w=\left\{x_{n}\right\}_{n=0,1, \ldots} \mid x_{i} \in \mathfrak{A}, A_{x_{i}, x_{i+1}}=1\right\}
$$

We assume further that the matrix $A$ has the property that $A_{i i}=1$ for each $i=0, \ldots, N-1$, that is, that arbitrarily long strings made of the same letters are allowed in the words of $\Lambda_{A}$.

The set $\Lambda_{A}$ can be topologized as a Cantor set, for example by identifying it with the subset of the interval $[0,1]$ of numbers whose base $N$ expansion satisfies the admissibility condition. However, notice that, when we choose to view $\Lambda_{A}$ as a subset of the interval $[0,1]$, which is convenient in what follows, we identify the rational numbers with infinite periodic sequences rather than with a finite $N$-adic expansion, so as to be able to act with the shift map $\sigma$ on all of $\Lambda_{A}$. More precisely, the map between the abstract set $\Lambda_{A}$ and its image inside the interval $[0,1]$ is two-to-one on the periodic sequences. These are of measure zero in the interval, so for our measure theoretic argument we ignore the distinction and use the same notation for both sets.

Let $\delta_{A}$ be the Hausdorff dimension of the set $\Lambda_{A}$, realized as a subset of the interval $[0,1]$ in this way. We can then consider the Hilbert space $L^{2}\left(\Lambda_{A}, \mu_{A}\right)$, where $\mu_{A}$ is the Hausdorff measure in the dimension $\delta_{A}$.

We consider on $\Lambda_{A}$ the self-map given by the one-sided shift

$$
\sigma: \Lambda_{A} \rightarrow \Lambda_{A}, \quad \sigma\left(x_{0} x_{1} x_{2} \ldots x_{n} \ldots\right)=x_{1} x_{2} \ldots x_{n} \ldots
$$

Proposition 2.6 The shift $\sigma$ is the coding map of the semibranching function system

$$
\sigma_{i}: D_{i} \rightarrow R_{i}, \quad \sigma_{i}(w)=i w,
$$

where

$$
D_{i}=\left\{w=\left\{x_{k}\right\} \in \Lambda_{A} \mid A_{i, x_{0}}=1\right\}
$$

and

$$
R_{i}=\left\{w=\left\{x_{k}\right\} \in \Lambda_{A} \mid x_{0}=i\right\}=: \Lambda_{A}(i) .
$$

Proof We show that the maps of (2.12) form a semibranching function system. We have

$$
\Lambda_{A}=\cup_{i} R_{i}, \quad \text { with } \quad R_{i} \cap R_{j}=\emptyset, \quad i \neq j,
$$

hence the condition (2.3) of a semibranching function system is satisfied. Moreover, the Radon-Nikodym derivative 


$$
\Phi_{\sigma_{i}}=\frac{d \mu_{A} \circ \sigma_{i}}{d \mu_{A}}
$$

is well defined and positive, since the map $\sigma_{i}$ on $\Lambda_{A} \subset[0,1]$ is realized by contractions and translations. In fact, we can write the domain $D_{i}$ of the map $\sigma_{i}$ as

$$
D_{i}=\cup_{j: A_{i j}=1} R_{j}
$$

On each $R_{j}$ the map $\sigma_{i}$ is the restriction of the map of the $I_{j} \subset[0,1]$,

$$
I_{j}=\left\{w \in[0,1] \mid x_{0}=j\right\},
$$

where $x_{0}$ is the first digit in the $N$-adic expansion of $w=0 . x_{0} x_{1} x_{2} \ldots$, that maps it to the subset $I_{j j}$ of elements with first and second digit equal to $j$ composed with a translation that maps isometrically $I_{j j} \rightarrow I_{i j}$ to the interval of all numbers with first digit $i$ and second digit $j$. It is then clear that the shift map (2.11) is a coding map for this semibranching function system, since on each $D_{i}$ we have $\sigma \circ \sigma_{i}(w)=w$.

One then sees easily that this gives a representation of the Cuntz-Krieger algebra $O_{A}$ of the type described in Proposition 2.5 above.

Proposition 2.7 The operators $T_{i}$ and $T_{i}^{*}$ of (2.4) and (2.5) acting on $\mathcal{H}_{A}=$ $L^{2}\left(\Lambda_{A}, \mu_{A}\right)$ define a representation of $O_{A}$ with generators $S_{i}=T_{i}$.

Proof The result immediately follows from Proposition 2.5, upon noticing that the condition (2.15) is the needed relation (2.8). We are assuming $A_{i i}=1$ for all $i$, so the hypothesis of Proposition 2.5 are satisfied.

It is well known (see [9]) that the abelian $C^{*}$-algebra $C\left(\Lambda_{A}\right)$ sits naturally inside the Cuntz-Krieger algebra $O_{A}$ as the $C^{*}$-subalgebra generated by the range projections

$$
S_{x_{1}} \cdots S_{x_{n}} S_{x_{n}}^{*} \cdots S_{x_{1}}^{*}
$$

for arbitrary $x_{i} \in \mathfrak{A}$ and arbitrary $n$.

\subsection{Perron-Frobenius Operator}

Consider the operator $T_{\sigma}: L^{2}(X, \mu) \rightarrow L^{2}(X, \mu)$ that composes with the coding $\operatorname{map} \sigma: X \rightarrow X$,

$$
\left(T_{\sigma} \psi\right)(x)=\psi(\sigma(x))
$$

It is well known in the theory of dynamical systems that one can associate to a self map $\sigma: X \rightarrow X$ of a measure space its Perron-Frobenius operator $\mathcal{P}_{\sigma}$. This is defined as the adjoint of the composition (2.16) by

$$
\int \bar{\psi} \mathcal{P}_{\sigma}(\xi) d \mu=\int \overline{T_{\sigma}(\psi)} \xi d \mu .
$$


Proposition 2.8 Let $\left\{\sigma_{i}\right\}_{i=1}^{N}$ be a semibranching function system with coding map $\sigma: X \rightarrow X$. Then the Perron-Frobenius operator $\mathcal{P}_{\sigma}$ is of the form

$$
\left(\mathcal{P}_{\sigma} \xi\right)(x)=\sum_{i} \chi_{D_{i}}(x) \Phi_{\sigma_{i}}(x) \xi\left(\sigma_{i}(x)\right)
$$

Proof In the inner product of $\mathcal{H}=L^{2}(X, \mu)$ we find

$$
\begin{aligned}
\langle T \psi, \xi\rangle & =\int_{X} \overline{\psi(\sigma(x))} \xi(x) d \mu(x) \\
& =\sum_{i} \int_{D_{i}} \overline{\psi(u)} \xi\left(\sigma_{i}(u)\right) \frac{d\left(\mu \circ \sigma_{i}\right)}{d \mu} d \mu(u)=\left\langle\psi, \sum_{i} \chi_{D_{i}} \Phi_{\sigma_{i}} \xi \circ \sigma_{i}\right\rangle .
\end{aligned}
$$

Notice the similarity of the Perron-Frobenius operator $\mathcal{P}_{\sigma}$ to the operators $T_{i}^{*}$ of (2.5) above. In fact, using (2.5) and Proposition 2.8, we easily get the following, which was observed already in [20].

Corollary 2.9 Let $\left\{\sigma_{i}\right\}_{i=1}^{N}$ be a semibranching function system with coding map $\sigma$ : $X \rightarrow X$. Then the Perron-Frobenius operator $\mathcal{P}_{\sigma}$ is of the form

$$
\mathcal{P}_{\sigma}=\sum_{i} \Phi_{\sigma_{i}}^{1 / 2} T_{i}^{*} .
$$

Notice that, in some particular cases, the functions $\Phi_{\sigma_{i}}$ may be constant, in which case (2.19) gives just a linear combination of the operators $T_{i}^{*}$. For example, in the cases considered in $[6,7]$ the functions $\Phi_{\sigma_{i}}$ are locally constant, while they are not in the case considered in [5].

In the case of representations as in Proposition 2.5, we can express the PerronFrobenius operator in terms of the partial isometries $S_{i}$ in the following way.

Proposition 2.10 Let $\left\{\sigma_{i}\right\}$ be a semibranching function system on $X$, where the $\sigma_{i}$ are defined on subsets $D_{i} \subset X$ satisfying (2.8) (possibly up to sets of measure zero). Then the Perron-Frobenius operator $\mathcal{P}_{\sigma}$ is a function of the adjoints $S_{i}^{*}$ of the generators of the Cuntz-Krieger algebra $O_{A}$ and the multiplication operators by the functions $\Phi_{\sigma_{i}}^{1 / 2}$ by

$$
\mathcal{P}_{\sigma}=\sum_{i} \Phi_{\sigma_{i}}^{1 / 2} S_{i}^{*}
$$

In the case where the $\Phi_{\sigma_{i}}^{1 / 2}$ are constant over $D_{i}$, the operator $\mathcal{P}_{\sigma}$ belongs to the algebra $O_{A}$. 
Proof The hypothesis are the same as in Proposition 2.5, hence we know that the generators $S_{i}$ of the Cuntz-Krieger algebra $O_{A}$ in the representation on $L^{2}(X, \mu)$ are given by the $T_{i}$ of (2.4). Then (2.19) gives (2.20). The case where the $\Phi_{\sigma_{i}}^{1 / 2}$ are constant over $D_{i}$ then follows immediately from (2.20), since $\mathcal{P}_{\sigma}$ is then a linear combination of the $S_{i}^{*}$.

To avoid having to assume that the $\Phi_{\sigma_{i}}$ are constant in the result above (although this will in fact be the case in the main example we will be considering later), one can more conveniently work with representations of the Cuntz-Krieger algebras on the Hilbert space of half-densities, analogous to the representations of the Cuntz algebra considered in [16].

Recall that the Hilbert space $\tilde{\mathcal{H}}$ of half densities consists of elements of the form $\psi(d \mu / d \lambda)^{1 / 2}$, where $\psi \in L^{2}(X, d \mu)$ and $\mu<<\lambda$ with $d \mu / d \lambda$ the Radon-Nikodym derivative, which $\lambda$-a.e. positive. Elements are considered modulo $\lambda$-a.e. equivalence and the inner product is given by

$$
\left\langle\psi\left(\frac{d \mu}{d \lambda}\right)^{1 / 2}, h\left(\frac{d \nu}{d \lambda}\right)^{1 / 2}\right\rangle=\int_{X} \bar{f}\left(\frac{d \mu}{d \lambda}\right)^{1 / 2} h\left(\frac{d \nu}{d \lambda}\right)^{1 / 2} d \lambda .
$$

One often writes elements of $\tilde{\mathcal{H}}$ with the notation $\psi \sqrt{d \mu}$.

Given a semibranching function system on $X$ satisfying (2.8), we can construct representations of the Cuntz-Krieger algebra $O_{A}$ on the space of half-densities of $X$, in much the same way as we did in Proposition 2.7 on the space $L^{2}(X, d \mu)$.

Proposition 2.11 Let $\left\{\sigma_{i}\right\}$ be a semibranching function system on $X$, where the $\sigma_{i}$ are defined on subsets $D_{i} \subset X$ satisfying (2.8), possibly up to sets of measure zero. Let $\tilde{\mathcal{H}}$ be the Hilbert space of half-densities on X. Consider the operators

$$
\tilde{S}_{i}(\psi \sqrt{d \mu})=\chi_{R_{i}}(\psi \circ \sigma) \sqrt{d \mu \circ \sigma} .
$$

These operators define a representation of the Cuntz-Krieger algebra $O_{A}$.

Proof To compute the adjoints $S_{i}^{*}$ we check

$$
\begin{aligned}
& \left\langle\tilde{S}_{i}(\psi \sqrt{d \mu}), \xi \sqrt{\nu}\right\rangle=\int_{R_{i}} \overline{\psi(\sigma(x))} \xi(x)\left(\frac{d \mu(\sigma(x))}{d \lambda}\right)^{1 / 2}\left(\frac{d \nu(x)}{d \lambda}\right)^{1 / 2} d \lambda(x) \\
& =\int_{D_{i}} \overline{\psi(u)} \xi\left(\sigma_{i}(u)\right)\left(\frac{d \mu(u)}{d \lambda \circ \sigma_{i}}\right)^{1 / 2}\left(\frac{d \nu\left(\sigma_{i}(u)\right)}{d \lambda \circ \sigma_{i}}\right)^{1 / 2} \frac{d \lambda \circ \sigma_{i}(u)}{d \lambda} d \lambda(u) \\
& =\int_{D_{i}} \overline{\psi(u)} \xi\left(\sigma_{i}(u)\right)\left(\frac{d \mu(u)}{d \lambda}\right)^{1 / 2}\left(\frac{d \nu\left(\sigma_{i}(u)\right)}{d \lambda}\right)^{1 / 2} d \lambda(u) \\
& =\left\langle\psi \sqrt{d \mu}, \chi_{D_{i}} \xi \circ \sigma_{i} \sqrt{d \nu \circ \sigma_{i}}\right\rangle,
\end{aligned}
$$


which gives

$$
\tilde{S}_{i}^{*}(\xi \sqrt{d \nu})=\chi_{D_{i}}\left(\xi \circ \sigma_{i}\right) \sqrt{d \nu \circ \sigma_{i}}
$$

We then check that the operators $\tilde{S}_{i}$ and $\tilde{S}_{i}^{*}$ satisfy the Cuntz-Krieger relations (2.1) and (2.2). We have

$$
\begin{aligned}
\left\langle\tilde{S}_{i}^{*}(\xi \sqrt{d \nu}), \tilde{S}_{i}(\xi \sqrt{d \nu})\right\rangle & =\int_{D_{i}}\left|\xi\left(\sigma_{i}(x)\right)\right|^{2} \frac{d \nu \circ \sigma_{i}}{d \lambda} d \lambda(x) \\
& =\int_{R_{i}}|\xi(u)|^{2} \frac{d \nu}{d \lambda \circ \sigma} \frac{d \lambda \circ \sigma}{d \lambda} d \lambda(u)=\int_{R_{i}}|\xi|^{2} \frac{d \nu}{d \lambda} d \lambda
\end{aligned}
$$

which shows that $\tilde{S}_{i} \tilde{S}_{i}^{*}=\tilde{P}_{i}$, the range projection given by multiplication by the characteristic function of $R_{i}$, so that the relation (2.2) is satisfied by the orthogonality of the projections $\tilde{P}_{i}$

$$
\sum_{i} \tilde{S}_{i} \tilde{S}_{i}^{*}=1
$$

We also have

$$
\begin{aligned}
\left\langle\tilde{S}_{i}(\psi \sqrt{d \mu}), \tilde{S}_{i}(\psi \sqrt{d \mu})\right\rangle & =\int_{R_{i}}|\psi(\sigma(x))|^{2} \frac{d \mu \circ \sigma}{d \lambda} d \lambda(x) \\
& =\int_{D_{i}}|\psi(u)|^{2} \frac{d \mu}{d \lambda \circ \sigma_{i}} \frac{d \lambda \circ \sigma_{i}}{d \lambda} d \lambda(u)=\int_{D_{i}}|\psi(u)|^{2} \frac{d \mu}{d \lambda} d \lambda(u),
\end{aligned}
$$

which shows that $\tilde{S}_{i}^{*} \tilde{S}_{i}=\tilde{Q}_{i}$, where $\tilde{Q}_{i}$ is the domain projection given by multiplication by the characteristic function of $D_{i}$. Using the relation (2.8) this then gives

$$
\tilde{S}_{i}^{*} \tilde{S}_{i}=\sum_{j} A_{i j} \tilde{S}_{i} \tilde{S}_{i}^{*},
$$

which shows that (2.1) is satisfied.

We then compute explicitly the Perron-Frobenius operator of the coding map $\sigma$ : $X \rightarrow X$ acting on the space of half-densities.

Proposition 2.12 Let $\sigma: X \rightarrow X$ be the coding map of a semibranching function system as in Proposition 2.11 above. The Perron-Frobenius operator $\tilde{\mathcal{P}}_{\sigma}$ on the Hilbert space of half-densities is given by

$$
\tilde{\mathcal{P}}_{\sigma}=\sum_{i} \tilde{S}_{i}^{*},
$$


where $\tilde{S}_{i}$ are the generators (2.22) of the representation of the Cuntz-Krieger algebra $O_{A}$ on $\tilde{\mathcal{H}}$.

Proof The translation operator associated to the shift map $\sigma: X \rightarrow X$ is acting on the space of half-densities by

$$
\tilde{T}_{\sigma}(\psi \sqrt{d \mu})=\psi \circ \sigma \sqrt{d \mu \circ \sigma}
$$

The Perron-Frobenius operator $\tilde{\mathcal{P}}_{\sigma}$ on $\tilde{\mathcal{H}}$ is the adjoint

$$
\left\langle\tilde{T}_{\sigma}(\psi \sqrt{d \mu}), \xi \sqrt{d \nu}\right\rangle=\left\langle\psi \sqrt{d \mu}, \tilde{\mathcal{P}}_{\sigma}(\xi \sqrt{d \nu})\right\rangle
$$

This gives

$$
\begin{aligned}
& \int_{X} \overline{\psi(\sigma(x))}\left(\frac{d \mu \circ \sigma}{d \lambda}\right)^{1 / 2} \xi(x)\left(\frac{d \nu}{d \lambda}\right)^{1 / 2} d \lambda(x) \\
& \quad=\sum_{i} \int_{R_{i}} \overline{\psi(u)}\left(\frac{d \mu}{d \lambda \circ \sigma_{i}}\right)^{1 / 2} \xi\left(\sigma_{i}(u)\right)\left(\frac{d \nu \circ \sigma_{i}}{d \lambda \circ \sigma_{i}}\right)^{1 / 2} \frac{d \lambda \circ \sigma_{i}}{d \lambda} d \lambda(u) \\
& =\sum_{i} \int_{R_{i}} \overline{\psi(u)}\left(\frac{d \mu}{d \lambda}\right)^{1 / 2} \xi\left(\sigma_{i}(u)\right)\left(\frac{d \nu \circ \sigma_{i}}{d \lambda}\right)^{1 / 2} d \lambda(u)
\end{aligned}
$$

which gives

$$
\tilde{\mathcal{P}}_{\sigma}(\xi \sqrt{d \nu})=\sum_{i} \chi_{R_{i}}\left(\xi \circ \sigma_{i}\right) \sqrt{d \nu \circ \sigma_{i}}
$$

which is (2.24).

For example, in the case of the Cuntz-Krieger algebras considered in [5-7], where the representation comes from the action of a Schottky group $\Gamma$ on its limit set, the generators $S_{i}$ are associated to a symmetric set of generators $\mathfrak{A}=\left\{\gamma_{1}, \ldots, \gamma_{g}, \gamma_{1}^{-1}, \ldots\right.$, $\left.\gamma_{g}^{-1}\right\}$ of a Schottky group of genus $g$, and the matrix $A$ of the Cuntz-Krieger algebra has $A_{i j}=1$ for $|i-j| \neq g$ and zero otherwise, corresponding to the admissibility of the infinite sequences $w=a_{0} a_{1} a_{2} \cdots$ of elements of $\mathfrak{A}$ parameterizing points in the limit set $\Lambda_{\Gamma}$, namely that $a_{i+1} \neq a_{i}^{-1}$. In this particular class of examples, the Perron-Frobenius operator of Proposition 2.12 has the form

$$
\tilde{\mathcal{P}}_{\sigma}=\tilde{S}_{\gamma_{1}}^{*}+\tilde{S}_{\gamma_{1}^{-1}}^{*}+\cdots+\tilde{S}_{\gamma_{g}}^{*}+\tilde{S}_{\gamma_{g}^{-1}}^{*}
$$

This resembles closely a Harper operator for the group $\Gamma$, save for the important difference that the operators associated to the symmetric set of generators of $\Gamma$ here are partial isometries and not unitaries as in the usual Harper operator. 


\subsection{Projection Valued Measures}

We recall how one constructs projection-valued measures using subdivions of compact metric spaces and subdivisions of projections in Hilbert spaces. (We follow the notation and terminology of [18] for the standard material we recall.) We then show how this technique applies to the representations of Cuntz-Krieger algebras described above.

We begin by recalling the notion of partitions and $N$-adic systems of partitions of a metric space.

Definition 2.13 Let $(X, d)$ be a compact metric space. For subsets $A \subset X$, define the diameter as

$$
|A|:=\sup \{d(x, y) \mid x, y \in A\} .
$$

A partition $\mathcal{P}$ of $X$ is a family $\{A(i)\}_{i \in I}$, for a (finite) index set $I$, with the property that

(1) $\bigcup_{i} A(i)=X$.

(2) $A(i) \cap A(j)=\emptyset$, for $i \neq j$.

For a given $N \geq 2$, an $N$-adic system of partitions of $X$ is a family (indexed by $k \in \mathbb{N}$ ) of partitions $\mathcal{P}_{k}$ of $X$ into Borel subsets $A_{k}(a)$, indexed by elements of $\mathfrak{A}^{k}$, where $\mathfrak{A}=\{0, \ldots, N-1\}$ is the given alphabet on $N$ letters, with the properties:

(1) $\left|A_{k}(a)\right|=O\left(N^{-c k}\right)$, for some $c>0$.

(2) Every $A_{k+1}(b)$, with $b \in \mathfrak{A}^{k+1}$, is contained in some $A_{k}(a)$, for some $a \in \mathfrak{A}^{k}$.

We then recall the equally well known notion of partitions of projections in Hilbert spaces.

Definition 2.14 Let $\mathcal{H}$ be a complex separable Hilbert space. A partition of projections in $\mathcal{H}$ is a collection $\{P(i)\}_{i \in I}$ of projections $P(i)=P(i)^{*}=P(i)^{2}$ such that

(1) $P(i) P(j)=0$, for $i \neq j$.

(2) $\sum_{i} P(i)=1$.

An $N$-adic system of partitions of $\mathcal{H}$ into projections is a family of partitions into projections $\left\{P_{k}(a)\right\}$ indexed by $a \in \mathfrak{A}^{k}$ such that, for every $P_{k+1}(a)$, there is some $b \in \mathfrak{A}^{k}$ with $P_{k}(b) P_{k+1}(a)=P_{k+1}(a)$.

We also recall the notion of operator valued measure.

Definition 2.15 Denote by $\mathcal{B}(X)$ the collection of Borel subsets of a compact metric space $X$. A positive operator-valued function $E: \mathcal{B}(X) \rightarrow \mathcal{L}(\mathcal{H})$ defined on $\mathcal{B}(X)$ with values in bounded linear operators on a Hilbert space $\mathcal{H}$ is called a $\sigma$ additive measure if, given a sequence $B_{1}, B_{2} \ldots$, in $\mathcal{B}(X)$, such that $B_{i} \cap B_{j}=\emptyset$ for $i \neq j$, one has

$$
E\left(\bigcup_{i} B_{i}\right)=\sum_{i} E\left(B_{i}\right)
$$


An orthogonal projection valued measure is a positive operator-valued measure as above satisfying:

(1) $E(B)=E(B)^{*}=E(B)^{2}$, for all $B \in \mathcal{B}(X)$.

(2) $E\left(B_{1}\right) E\left(B_{2}\right)=0$ when $B_{1} \cap B_{2}=\emptyset$.

(3) $E(X)=1$, the identity on $\mathcal{H}$.

Note that the values $E\left(B_{i}\right)$ in (2.26) are positive operators, so we take the summation on the right hand side of (2.26) to be convergent in the strong operator topology.

We are interested here in a particular construction of $N$-adic partitions, for the metric Cantor set $\Lambda_{A}$ defined in (2.10) above. As above, we consider the alphabet $\mathfrak{A}=\{0, \ldots, N-1\}$. For any $k \in \mathbb{N}$, we denote by $\mathcal{W}_{k, A} \subset \mathfrak{A}^{k}$ the finite set of all admissible words of length $k$ in the alphabet $\mathfrak{A}$,

$$
\mathcal{W}_{k, A}=\left\{a=\left(a_{1}, \ldots, a_{k}\right) \in \mathfrak{A}^{k} \mid A_{a_{i}, a_{i+1}}=1, i=1, \ldots, k\right\}
$$

We also denote by $\Lambda_{k, A}(a)$ the clopen subset of the Cantor set $\Lambda_{A}$ given by all words that start with a given $a \in \mathcal{W}_{k, A}$,

$$
\Lambda_{k, A}(a)=\left\{w=\left(w_{1}, w_{2}, \ldots, w_{n}, \ldots\right) \in \Lambda_{A} \mid\left(w_{1}, \ldots, w_{k}\right)=a\right\}
$$

We then have the following partition and corresponding operator valued measure.

Proposition 2.16 The subsets $\Lambda_{k, A}(a)$ of (2.28) define an $N$-adic system of partitions for $\Lambda_{A}$. There is a corresponding $N$-adic system of projections $P_{k}(a)$ on the Hilbert space $\mathcal{H}=L^{2}\left(\Lambda_{A}, \mu_{A}\right)$ and an orthogonal projection valued measure $E$ on $\mathcal{B}\left(\Lambda_{A}\right)$ satisfying

$$
E\left(\Lambda_{k, A}(a)\right)=P_{k}(a)
$$

for all $k \in \mathbb{N}$ and for all $a \in \mathcal{W}_{k, A}$.

Proof To see that the $\Lambda_{k, A}(a)$ form an $N$-adic system of partitions, notice that, when we identify $\Lambda_{A}$ with the subset of $[0,1]$ of numbers with admissible $N$-adic digital expansion and we measure diameters in the Euclidean distance on $[0,1]$, we see that the set $\Lambda_{k, A}(a)$, which consists of such numbers with fixed first $k$ digits in the $N$-adic expansion have

$$
\left|\Lambda_{k, A}(a)\right| \leq N^{-k}
$$

since the sets of all numbers with fixed $k$ digits in the $N$-adic expansion are intervals of length $N^{-k}$. Moreover, by construction we have inclusions

$$
\Lambda_{k, A}\left(a_{1}, \ldots, a_{k}\right) \subset \Lambda_{k-1, A}\left(a_{1}, \ldots, a_{k-1}\right) .
$$

We also have, for fixed $k$,

$$
\Lambda_{k, A}(a) \cap \Lambda_{k, A}(b)=\emptyset, \quad \text { for } a \neq b \in \mathcal{W}_{k, A},
$$


and

$$
\cup_{a \in \mathcal{W}_{k, A}} \Lambda_{k, A}(a)=\Lambda_{A}
$$

Thus, we have an $N$-adic system of partitions.

One knows from [9] that there is an $*$-isomorphism between the $C^{*}$-algebra of continuous functions $C\left(\Lambda_{A}\right)$ and the maximal abelian subalgebra of the Cuntz-Krieger algebra $O_{A}$ generated by all the range projections

$$
P_{k}(a)=S_{a_{1}} \cdots S_{a_{k}} S_{a_{k}}^{*} \cdots S_{a_{1}}^{*} .
$$

We show that the $P_{k}(a)$ define an $N$-adic system of projections on the Hilbert space $\mathcal{H}=L^{2}\left(\Lambda_{A}, \mu_{A}\right)$. In the representation of $O_{A}$ described in Proposition 2.5, the operator $P_{k}(a)$ acts as the projection given by multiplication by the characteristic function of the set $\Lambda_{k, A}(a)$.

Since the $\Lambda_{k, A}(a)$ form an $N$-adic system of partitions, in particular, as we have seen above, there are inclusions (2.31). These imply that the corresponding projections satisfy

$$
P_{k-1}\left(a_{1}, \ldots, a_{k-1}\right) P_{k}\left(a_{1}, \ldots, a_{k}\right)=P_{k}\left(a_{1}, \ldots, a_{k}\right) .
$$

More precisely, one can see by writing as in (2.32) and using (2.2) that

$$
\begin{aligned}
\sum_{a_{k} \in \mathfrak{A}} P_{k}\left(a_{1}, \ldots, a_{k}\right) & =\sum_{a_{k} \in \mathfrak{A}} S_{a_{1}} \cdots S_{a_{k}} S_{a_{k}}^{*} \cdots S_{a_{1}}^{*} \\
& =S_{a_{1}} \cdots S_{a_{k-1}}\left(\sum_{a_{k} \in \mathfrak{A}} S_{a_{k}} S_{a_{k}}^{*}\right) S_{a_{k-1}}^{*} \cdots S_{a_{1}}^{*} \\
& =S_{a_{1}} \cdots S_{a_{k-1}} S_{a_{k-1}}^{*} \cdots S_{a_{1}}^{*}=P_{k-1}\left(a_{1}, \ldots, a_{k-1}\right) .
\end{aligned}
$$

For every $k \in \mathbf{Z}_{+}$, let us denote by $\mathcal{U}_{k}$ the finite dimensional subalgebra of $C\left(\Lambda_{A}\right)$ spanned by the finite linear combinations

$$
\sum_{a \in \mathcal{W}_{k, A}} c_{a} \chi_{\Lambda_{k, A}(a)}
$$

The inclusions (2.31) determine embeddings $\mathcal{U}_{k-1} \rightarrow \mathcal{U}_{k}$ and the bound (2.30) on the diameters implies that every function in $C\left(\Lambda_{A}\right)$ can be uniformly approximated with a sequence of functions in $\mathcal{U}=\lim _{k} \mathcal{U}_{k}$. Thus, the homomorphism

$$
\pi: \sum_{a \in \mathcal{W}_{k, A}} c_{a} \chi_{\Lambda_{k, A}(a)} \mapsto \sum_{a \in \mathcal{W}_{k, A}} c_{a} P_{k}(a)
$$

extends, by a standard argument from function theory, from $C\left(\Lambda_{A}\right)$ to all the Baire functions on $\Lambda_{A}$. 
It makes sense then to define an operator valued measure by setting

$$
E(B):=\pi\left(\chi_{B}\right)
$$

where we still denote as $\pi$ the extension above. It follows that $E(\cdot)$ satisfies the properties of Definition 2.15 and is countably additive. It also satisfies $E\left(\Lambda_{k, A}(a)\right)=P_{k}(a)$, for every $k \in \mathbf{Z}_{+}$and for all $a \in \mathcal{W}_{k, A}$.

\subsection{Hausdorff Dimension}

We consider again the space $\Lambda_{A}$ of numbers in the interval $[0,1]$ whose $N$-adic expansion is admissible according to the matrix $A$, that is, $x=0 . a_{0} a_{1} \cdots a_{n} \cdots$ with $A_{a_{i}, a_{i+1}}=1$.

We know that in this case the maps $\sigma_{i}$ are defined on domains $D_{i} \subset \Lambda_{A}$ satisfying $D_{i}=\cup_{j: A_{i j}=1} R_{j}$, where $R_{j} \subset \Lambda_{A}$ is the range of $\sigma_{j}$, with $\Lambda_{A}=\cup_{j} R_{j}$ and $R_{i} \cap R_{j}=\emptyset$ when $i \neq j$. We then have the following properties.

Theorem 2.17 Assume that the non-negative matrix $A$ is irreducible, that is, there exists a power $A^{n}$ for which all entries are positive. Let $\delta_{A}$ be the Hausdorff dimension of $\Lambda_{A}$ and $\mu_{A}=\mu_{\mathcal{H}, \delta_{A}}$ the corresponding Hausdorff measure.

(1) On the sets $D_{i} \subset \Lambda_{A}$, the Radon-Nikodym derivatives are constant and equal to

$$
\Phi_{\sigma_{i}}=\frac{d \mu \circ \sigma_{i}}{d \mu}=N^{-\delta_{A}}
$$

(2) The Hausdorff measure $\mu=\mu_{A}$ on $\Lambda_{A}$ satisfies

$$
\mu\left(R_{i}\right)=p_{i}
$$

where $p=\left(p_{i}\right)_{i=0, \ldots, N-1}$ is the Perron-Frobenius eigenvector of the matrix $A$,

$$
\sum_{j} A_{i j} p_{j}=r(A) p_{i},
$$

with eigenvalue the spectral radius $r(A)$, and normalized to have $\sum_{i} p_{i}=1$.

(3) The Hausdorff dimension of $\Lambda_{A}$ is given by

$$
\delta_{A}=\operatorname{dim}_{H}\left(\Lambda_{A}\right)=\frac{\log r(A)}{\log N},
$$

with $r(A)$ the spectral radius of the matrix $A$.

(4) The measure $\mu$ satisfies the self-similarity condition

$$
\mu=N^{-\delta_{A}} \sum_{k=0}^{n-1} \mu \circ \sigma_{k}^{-1},
$$

where $\mu\left(\sigma_{k}^{-1}(E)\right)=\mu\left(\left\{x \in \Lambda_{A} \mid \sigma_{k}(x) \in E\right\}\right)$. 
Proof (1) The maps $\sigma_{i}$ act as the restrictions to the set $D_{i}$ of the linear maps

$$
\sigma_{i}(x)=\frac{x+i}{N}
$$

defined on the interval $[0,1]$. Thus, we see directly that the Radon-Nikodym derivative of the Hausdorff measure $\mu_{\mathcal{H}, s}$ will give

$$
\frac{d \mu_{\mathcal{H}, s} \circ \sigma_{i}}{d \mu_{\mathcal{H}, s}}=N^{-s}
$$

In particular for $s=\delta_{A}=\operatorname{dim}_{H}\left(\Lambda_{A}\right)$ this gives (2.35).

(2) We first show that setting

$$
v\left(R_{i}\right)=p_{i},
$$

with $p$ the normalized Perron-Frobenius eigenvector of $A$, defines a probability measure on $\Lambda_{A}$.

The Perron-Frobenius theorem for the matrix $A$ shows that, if $r(A)$ denotes the spectral radius of $A$, then $r(A)$ is an eigenvalue which has an eigenvector $p=\left(p_{i}\right)$ with non-negative entries. We can normalize it so that $\sum_{i} p_{i}=1$. Setting $v\left(R_{i}\right)=p_{i}$ defines a measure on $\Lambda_{A}$. In fact, it suffices to see that we can define $v\left(\Lambda_{k, A}(a)\right)$ compatibly, for all $a \in \mathcal{W}_{k, A}$. We set

$$
v\left(\Lambda_{k, A}(a)\right)=r(A)^{-k} p_{a_{k}}
$$

where $a=\left(a_{1}, \ldots, a_{k}\right) \in \mathcal{W}_{k, A}$. To see that (2.42) consistently defines a measure on $\Lambda_{A}$ we need to check that

$$
v\left(\Lambda_{k, A}(a)\right)=\sum_{j=0}^{N-1} A_{a_{k} j} v\left(\Lambda_{k+1, A}(a j)\right)
$$

We have

$$
\sum_{j} A_{a_{k} j} v\left(\Lambda_{k+1, A}(a j)\right)=\sum_{j} A_{a_{k} j} r(A)^{-k-1} p_{j}=r(A)^{-k} p_{a_{k}}=v\left(\Lambda_{k, A}(a)\right),
$$

where we used the Perron-Frobenius relation

$$
p_{a_{k}}=r(A)^{-1} \sum_{j} A_{a_{k} j} p_{j} .
$$

The measure $v$ thus satisfies the self-similarity property

$$
v=r(A)^{-1} \sum_{j=0}^{n-1} v \circ \sigma_{j}^{-1}
$$


Indeed, it suffices to check it on sets of the form $E=\Lambda_{k, A}(a)$, for which $\sigma_{j}^{-1}\left(\Lambda_{k, A}(a)\right)$ is non-empty for $a_{1}=j$, in which case it is $\Lambda_{k-1, A}(\sigma(a))$. Then we have

$$
v\left(\Lambda_{k, A}(j b)\right)=r(A)^{-1} r(A)^{-|b|} p_{b_{k}}=r(A)^{-1} v\left(\Lambda_{k-1, A}(\sigma(a))\right),
$$

which gives (2.44).

We then compare this with the Hausdorff measure $\mu=\mu_{A}$. This satisfies

$$
\mu\left(R_{i}\right)=N^{-\delta_{A}} \sum_{j} A_{i j} \mu\left(R_{j}\right)
$$

In fact, this follows simply from the fact shown in (1) that the Radon-Nikodym derivatives are constant,

$$
\Phi_{\sigma_{i}}=\frac{d \mu \circ \sigma_{i}}{d \mu}=N^{-\delta}
$$

which gives

$$
\mu\left(R_{i}\right)=\int_{D_{i}} \frac{d \mu \circ \sigma_{i}}{d \mu} d \mu=N^{-\delta_{A}} \mu\left(D_{i}\right)=N^{-\delta_{A}} \sum_{j} A_{i j} \mu\left(R_{j}\right) .
$$

Note that it then follows that the measure $\mu$ also satisfies

$$
\mu\left(\Lambda_{k, A}(a)\right)=N^{-k \delta_{A}} \mu\left(R_{a_{k}}\right),
$$

for $a=\left(a_{1}, \ldots, a_{k}\right)$. This follows directly from (2.45) and the fact that

$$
\mu\left(\Lambda_{k, A}(a)\right)=\sum_{j} A_{a_{k} j} \mu\left(\Lambda_{k+1, A}(a j)\right) .
$$

Notice then that (2.45) is saying that the vector $q=\left(q_{i}\right)$ with $q_{i}=\mu\left(R_{i}\right)$ is also an eigenvector of the matrix $A$, with eigenvalue $N^{-\delta_{A}} \leq r(A)$, with the normalization $\sum_{i} q_{i}=1$.

Under the assumption that the non-negative matrix $A$ is irreducible, the PerronFrobenius theorem for $A$ ensures that the eigenvalue $r(A)$ is simple and that if $q=\left(q_{i}\right)$ is another eigenvector, $A q=\lambda q$ with $q_{i} \geq 0$, then $\lambda=r(A)$ and $q$ is a scalar multiple of $p$. Since both vectors are normalized, this implies that

$$
N^{\delta_{A}}=r(A) \quad \text { and } \quad v\left(R_{i}\right)=p_{i}=q_{i}=\mu\left(R_{i}\right) .
$$

By (2.46) and (2.42), this implies that the measures $\mu$ and $v$ agree.

(3) then follows immediately from $r(A)=N^{\delta_{A}}$ and (4) is just the self-similarity (2.44). 
As a particular case, if the matrix $A$ has the property that the value $\alpha=\sum_{j} A_{i j}$ is the same for all $i=0, \ldots, N-1$, then one has uniform probability for all the $R_{i}$, equal to $\mu\left(R_{i}\right)=1 / N$, and the set $\Lambda_{A}$ has then Hausdorff dimension $\delta_{A}=\log (\alpha) / \log (N)$.

We return to consider now in particular the representation of the Cuntz-Krieger algebra $O_{A}$ on the space $L^{2}\left(\Lambda_{A}, d \mu_{A}\right)$ as in Sect. 2.1.

Corollary 2.18 The Perron-Frobenius operator $\mathcal{P}_{\sigma}$ on the Hilbert space $L^{2}\left(\Lambda_{A}\right.$, $\left.d \mu_{A}\right)$, with $\mu_{A}=\mu_{\mathcal{H}, \delta_{A}}$ the Hausdorff measure with $\delta_{A}=\operatorname{dim}_{H}\left(\Lambda_{A}\right)$, satisfies

$$
\mathcal{P}_{\sigma}=N^{-\delta_{A} / 2} \sum_{i} S_{i}^{*}
$$

Proof As we have seen in Proposition 2.17, in this case the $\Phi_{\sigma_{i}}$ are locally constant and equal to $N^{-\delta_{A}}$, with $\delta_{A}$ the Hausdorff dimension, which in turn is given in terms of the spectral radius of $A$. Then we have from Proposition 2.10 that the Perron-Frobenius operator $\mathcal{P}_{\sigma}$ on $L^{2}\left(\Lambda_{A}, d \mu_{A}\right)$ is simply given by (2.48), where the $S_{i}$ generate the representation of the Cuntz-Krieger algebra on $L^{2}\left(\Lambda_{A}, d \mu_{A}\right)$.

We then see that one can use the result of Theorem 2.17 to construct a fixed point for the Perron-Frobenius operator $\mathcal{P}_{\sigma}$.

Proposition 2.19 Assume that the matrix $A$ is irreducible, and let $\omega$ be the PerronFrobenius eigenvector for $A^{t}$. Then $f=\sum_{i} \omega_{i} \chi_{R_{i}}$ is a fixed point of the PerronFrobenius operator $\mathcal{P}_{\sigma}$.

Proof Let $\omega$ be the Perron-Frobenius eigenvector

$$
A^{t} \omega=r(A) \omega \text {. }
$$

The Perron-Frobenius operator $\mathcal{P}_{\sigma}$ acting on the function $f=\sum_{i} \omega_{i} \chi_{R_{i}}$ gives

$$
\mathcal{P}_{\sigma}(f)=N^{-\delta_{A}} \sum_{i} \chi_{D_{i}} f \circ \sigma_{i}
$$

by Corollary 2.18. We have

$$
\chi_{R_{k}} \circ \sigma_{i}=\delta_{i k} \chi_{D_{i}}
$$

which gives

$$
\mathcal{P}_{\sigma}(f)=N^{-\delta_{A}} \sum_{i} \omega_{i} \chi_{D_{i}}=N^{-\delta_{A}} \sum_{i j} \omega_{i} A_{i j} \chi_{R_{j}}
$$

from (2.8). Using then $A^{t} \omega=r(A) \omega$ we obtain

$$
\mathcal{P}_{\sigma}(f)=N^{-\delta_{A}} r(A) \sum_{i} \omega_{i} \chi_{R_{i}}=\sum_{i} \omega_{i} \chi_{R_{i}}=f,
$$

where we used the fact that $r(A)=N^{\delta_{A}}$ as in (2.47). 
There is a well known relation for Cuntz-Krieger algebras between the fixed points of the dual Perron-Frobenius (or Ruelle transfer operator) acting on measures and KMS states with respect to associated time evolutions, see [22]. We discuss the more general case of the Ruelle transfer operators later, but we comment here on the case that follows directly from Theorem 2.17.

Corollary 2.20 On the Cuntz-Krieger algebra $O_{A}$ consider the time evolution defined by setting

$$
\sigma_{t}\left(S_{i}\right)=N^{i t} S_{i}
$$

The measure $\mu=\mu_{A}$ on $\Lambda_{A}$ defines a KMS state for the system $\left(O_{A}, \sigma_{t}\right)$ at inverse temperature $\beta=\delta_{A}$.

Proof We define a state $\varphi$ on $O_{A}$ associated to the measure $\mu$ by setting

$$
\varphi\left(S_{a} S_{b}^{*}\right)= \begin{cases}0 & a \neq b \\ \mu\left(\Lambda_{k, A}(a)\right) & a=b \in \mathcal{W}_{k, A}\end{cases}
$$

We use here the fact that all elements in $O_{A}$ can be approximated by linear combinations of elements of the form $S_{a} S_{b}^{*}$. We then need to check that the state $\varphi$ satisfies the KMS condition at inverse temperature $\beta=\delta_{A}$ for the time evolution (2.49). Because of the form of the state (2.50), and the fact that the measure $\mu$ satisfies (2.46), it suffices to check that

$$
\varphi\left(S_{i}^{*} S_{i}\right)=N^{\beta} \varphi\left(S_{i} S_{i}^{*}\right)
$$

This follows since we have

$$
\varphi\left(S_{i}^{*} S_{i}\right)=\sum_{j} A_{i j} \varphi\left(S_{j} S_{j}^{*}\right)=\sum_{j} A_{i j} \mu\left(R_{j}\right)=N^{\delta_{A}} \mu\left(R_{i}\right)=N^{\delta_{A}} \varphi\left(S_{i} S_{i}^{*}\right),
$$

using the fact that $p=\left(p_{i}\right)$ with $p_{i}=\mu\left(R_{i}\right)$ is the Perron-Frobenius eigenvector of the matrix $A$.

\subsection{Real Valued Measures and Fourier Transforms}

Given an element $f \in \mathcal{H}$ with norm $\|f\|=1$, one can define a real valued measure on $\Lambda_{A} \subset[0,1]$ by setting

$$
\mu_{f}(B):=\langle f, E(B) f\rangle
$$

with $E(B)$ an operator valued measure as in Sect. 2.3.

Since each such $\mu_{f}$ is a compactly supported measure on the real line, it makes sense to consider its Fourier transform

$$
\widehat{\mu_{f}}(t):=\int e^{i t x} d \mu_{f}(x)
$$


We then have the following result, which is analogous to the case of the Cuntz algebras $O_{n}$ discussed in [17].

Proposition 2.21 For every function $f \in \mathcal{H}=L^{2}\left(\Lambda_{A}, d \mu_{A}\right)$ with $\|f\|=1$, the measure $\mu_{f}(E)=\langle f, P(E) f\rangle$ satisfies

$$
\sum_{k=0}^{N-1} \int_{\Lambda_{A}} \psi \circ \sigma_{k} d \mu_{S_{k}^{*} f}=\int_{\Lambda_{A}} \psi d \mu_{f}
$$

The Fourier transform $\widehat{\mu}_{f}(t)$ satisfies

$$
\widehat{\mu}_{f}(t)=\sum_{k=0}^{N-1} e^{\frac{i t k}{N}} \widehat{\mu_{S_{k}^{*} f}}\left(\frac{t}{N}\right)
$$

Proof We have

$$
\sum_{k} \int_{\Lambda_{A}} \psi \circ \sigma_{k} d \mu_{S_{k}^{*} f}=\sum_{k}\left\langle S_{k}^{*} f, \pi\left(\chi_{D_{k}} \psi \circ \sigma_{k}\right) S_{k}^{*} f\right\rangle
$$

where $\pi$ denotes the embedding $\pi: C\left(\Lambda_{A}\right) \hookrightarrow O_{A}$, as in (2.33), which realizes $C\left(\Lambda_{A}\right)$ as an abelian $*$-subalgebra of $O_{A}$, with $\pi\left(\chi_{\Lambda_{k, A}(a)}\right)=S_{a} S_{a}^{*}$. In the algebra $O_{A}$ we have the relations

$$
\begin{aligned}
& \pi(f) S_{k}=S_{k} \pi\left(\chi_{D_{k}} f \circ \sigma_{k}\right), \\
& S_{k} \pi(f)=\pi(f \circ \sigma) S_{k}, \\
& \pi(f) S_{k}^{*}=S_{k}^{*} \pi(f \circ \sigma), \\
& S_{k}^{*} \pi(f)=\pi\left(\chi_{D_{k}} f \circ \sigma_{k}\right) S_{k}^{*} .
\end{aligned}
$$

Thus, we have $\pi\left(\chi_{D_{k}} \psi \circ \sigma_{k}\right) S_{k}^{*}=S_{k}^{*} \pi(\psi)$ and we write (2.55) as

$$
\sum_{k}\left\langle f, S_{k} S_{k}^{*} \pi(\psi) f\right\rangle=\sum_{k}\left\langle f, \pi\left(\chi_{R_{k}} \psi\right) f\right\rangle=\sum_{k} \int_{R_{k}} \psi d \mu_{f},
$$

which gives (2.53). We then proceed as in [17], and observe that (2.53), applied to $\psi(x)=e^{i t x}$, gives

$$
\sum_{k} \int e^{i t \frac{x+k}{N}} d \mu_{S_{k}^{*} f}(x)=\int e^{i t x} d \mu_{f}(x)
$$

which gives (2.54). 
We can equivalently see (2.53) as an immediate consequence of (2.39), since we have

$$
\begin{aligned}
\int \psi d \mu_{f} & =\langle f, \pi(\psi) f\rangle=\int \psi|f|^{2} d \mu \\
& =N^{-\delta} \sum_{j}\left\langle\chi_{D_{j}} f \circ \sigma_{j}, \pi\left(\psi \circ \sigma_{j}\right) \chi_{D_{j}} f \circ \sigma_{j}\right\rangle \\
& =\sum_{j}\left\langle S_{j}^{*} f, \pi\left(\psi \circ \sigma_{j}\right) S_{j}^{*} f\right\rangle=\int \psi \circ \sigma_{j} d \mu_{S_{j}^{*} f},
\end{aligned}
$$

with $S_{j}^{*} f=N^{-\delta / 2} \chi_{D_{j}} f \circ \sigma_{j}$.

Iterating the relation (2.54) one obtains

$$
\hat{\mu}_{f}(t)=\sum_{a \in \mathcal{W}_{k, A}} e^{i t x(a)} \hat{\mu}_{S_{a}^{*} f}\left(\frac{t}{N^{k}}\right),
$$

where for $a=\left(a_{1}, \ldots, a_{k}\right) \in \mathcal{W}_{k, A}$ we denote by $x(a)$ the expression

$$
x(a)=\frac{a_{1}}{N}+\frac{a_{2}}{N^{2}}+\cdots+\frac{a_{k}}{N^{k}} .
$$

As in [17], we then obtain an approximation of the measure $\mu_{f}$ with a family of combinations of Dirac measures in the following way.

Corollary 2.22 Let $\mu_{f}^{(k)}$ denote the measure

$$
\mu_{f}^{(k)}(E)=\sum_{a \in \mathcal{W}_{k, A}}\left\|S_{a}^{*} f\right\|^{2} \delta_{a}(E),
$$

where $\delta_{a}$ is the Dirac measure supported at the rational point $x(a)$ in $\Lambda_{A}$ whose terminating $N$-adic expansion is of the form (2.59), for

$$
a=\left(a_{1}, \ldots, a_{k}\right) \in \mathcal{W}_{k, A}
$$

The measures $\mu_{f}^{(k)}$ weakly converge to $\mu_{f}$, when considered as functionals on the space of integrable functions $\psi$ on the real line whose Fourier transform satisfies

$$
\int|t \hat{\psi}(t)| d t<\infty .
$$

Proof We show that, for all functions $\psi$ with (2.61), we have

$$
\lim _{k \rightarrow \infty} \int_{\Lambda_{A}} \psi d \mu_{f}^{(k)}=\int_{\Lambda_{A}} \psi d \mu_{f}
$$


Passing to Fourier transforms, we have

$$
\int \psi d \mu_{f}^{(k)}-\int \psi d \mu_{f}=\int \hat{\psi}(t)\left(\hat{\mu}_{f}^{(k)}(t)-\hat{\mu}_{f}(t)\right) \frac{d t}{2 \pi}
$$

The Fourier transform of $\mu_{f}^{(k)}$ is clearly of the form

$$
\hat{\mu}_{f}^{(k)}(t)=\sum_{a \in \mathcal{W}_{k, A}} e^{i t x(a)}\left\|S_{a}^{*} f\right\|^{2}
$$

with $x(a)$ as in (2.59), and one can estimate as in [17]

$$
\left|\hat{\mu}_{f}(t)-\hat{\mu}_{f}^{(k)}(t)\right| \leq|t| N^{-k}
$$

This gives

$$
\left|\int \psi d \mu_{f}^{(k)}-\int \psi d \mu_{f}\right| \leq \frac{N^{-k}}{2 \pi} \int|t \hat{\psi}(t)| d t
$$

which gives the weak convergence $\mu_{f}^{(k)} \rightarrow \mu_{f}$.

\subsection{Sierpinski Fractals}

There is another fractal object, besides the limit set $\Lambda_{A}$, that is naturally associated to an $N \times N$-matrix $A$ with entries in $\{0,1\}$. This is a Sierpinksi fractal constructed in the following way. Consider the square $\mathbb{S}=[0,1] \times[0,1]$ and write points $(x, y) \in \mathbb{S}$ in terms of the $N$-adic expansion

$$
(x, y)=\left(\frac{x_{1}}{N}+\frac{x_{2}}{N^{2}}+\cdots+\frac{x_{k}}{N^{k}}+\cdots, \frac{y_{1}}{N}+\frac{y_{2}}{N^{2}}+\cdots+\frac{y_{k}}{N^{k}}+\cdots\right),
$$

with $\left(x_{i}, y_{i}\right) \in\{0, \ldots, N-1\} \times\{0, \ldots, N-1\}=\mathfrak{A}^{2}$, for all $i \geq 1$. We then consider the subset $\mathbb{S}_{A} \subset \mathbb{S}$ given by

$$
\mathbb{S}_{A}=\left\{(x, y) \in S \mid A_{x_{i}, y_{i}}=1, \forall i \geq 1\right\} .
$$

This is a Sierpinski fractal whose iterative construction starts by subdividing the unit square $\mathbb{S}$ into the $N^{2}$ subsquares of size $N^{-2}$ consisting of points $(x, y)$ with first digits of the $N$-adic expansion equal to given $(i, j) \in \mathfrak{A}^{2}$. One then keeps among these only those for which $A_{i j}=1$. The procedure is then iterated by subdividing each of the remaining squares into $N^{2}$ subsquares of size $N^{-4}$ and keeping only those for which the same condition $A_{i j}=1$ is satisfied, and so on. At each step a square is of size 
$N^{-2 k}$ is replaced by $D$ squares of size $N^{-2(k+1)}$, where

$$
D=\sum_{i=0}^{N-1} d_{i}, \quad \text { with } \quad d_{i}=\#\left\{j \mid A_{i j}=1\right\} .
$$

These satisfy $d_{i} \leq N$ and $D \leq N^{2}$. Thus, the Hausdorff dimension of the Sierpinski fractal $\mathbb{S}_{A}$ is simply

$$
\operatorname{dim}_{H}\left(\mathbb{S}_{A}\right)=\frac{\log D}{2 \log N}
$$

One can then consider maps $\tau_{(i, j)}: \mathbb{S}_{A} \rightarrow \mathbb{S}_{A}$, for $(i, j)$ satisfying $A_{i j}=1$, given by

$$
\tau_{(i, j)}(x, y)=\left(\tau_{i}(x), \tau_{j}(y)\right)=\left(\frac{x+i}{N}, \frac{x+j}{N}\right) .
$$

Notice how, unlike the $\sigma_{i}$ acting on $\Lambda_{A}$ that we considered before, here the $\tau_{(i, j)}$ are everywhere defined on $\mathbb{S}_{A}$. Since we are only considering such maps for pairs $(i, j)$ with $A_{i j}=1$, it is clear that the image $\left(\tau_{i}(x), \tau_{j}(y)\right)$ is still a point in $\mathbb{S}_{A}$. The corresponding coding map $\tau: \mathbb{S}_{A} \rightarrow \mathbb{S}_{A}$ is given by

$$
\tau(x, y)=(\tau(x), \tau(y))=\left(0 . x_{2} \cdots x_{k} \cdots, 0 . y_{2} \cdots y_{k} \cdots\right),
$$

for $(x, y)=\left(0 . x_{1} x_{2} \cdots x_{k} \cdots, 0 . y_{1} y_{2} \cdots y_{k} \cdots\right)$.

Lemma 2.23 The semibranching function system $\left\{\tau_{(i, j)}\right\}$ for $(i, j) \in \mathfrak{A}^{2}$ with $A_{i j}=1$ determines a representation of the Cuntz algebra $O_{D}$ on the Hilbert space $L^{2}\left(\mathbb{S}_{A}, \mu\right)$, with $\mu$ the Hausdorff measure of dimension $\delta=\operatorname{dim}_{H}\left(\mathbb{S}_{A}\right)$ as in (2.64).

Proof Let $\Phi_{(i, j)}$ denote the Radon-Nikodym derivative of the measure $\mu$ with respect to composition by $\tau_{(i, j)}$. Since $\tau_{(i, j)}$ is of the form (2.65), we have

$$
\Phi_{(i, j)}(x, y)=\frac{d \mu \circ \tau_{(i, j)}}{d \mu}=N^{-2 \delta}=\frac{1}{D} .
$$

We consider the operators $S_{(i, j)}$ and $S_{(i, j)}^{*}$ defined as in the general case of a semibranching function system in the form

$$
S_{(i, j)} f=\chi_{R_{(i, j)}} \cdot\left(\Phi_{(i, j)} \circ \tau\right)^{-1 / 2} \cdot f \circ \tau,
$$

with $R_{i, j} \subset \mathbb{S}_{A}$ the range of $\tau_{(i, j)}$. The adjoint $S_{(i, j)}^{*}$ in the inner product of $L^{2}\left(\mathbb{S}_{A}, \mu\right)$ is given by

$$
\left\langle S_{(i, j)} f, h\right\rangle=N^{\delta} \int_{R_{(i, j)}} f \circ \tau h d \mu=N^{\delta} \int_{\mathbb{S}_{A}} f h \circ \tau_{(i, j)} \Phi_{i j} d \mu,
$$


so that we get

$$
S_{(i, j)}^{*} h=\Phi_{i j}^{1 / 2} h \circ \tau_{(i, j)}=N^{-\delta} h \circ \tau_{(i, j)} .
$$

Thus, one sees that

$$
S_{(i, j)}^{*} S_{(i, j)}=1, \quad \text { and } \quad \sum_{(i, j): A_{i j}=1} S_{(i, j)} S_{(i, j)}^{*}=1 \text {, }
$$

since $S_{(i, j)} S_{(i, j)}^{*}$ is the range projection given by multiplication by $\chi_{R_{(i, j)}}$. Thus, the $S_{(i, j)}$ generate a representation of the Cuntz algebra $O_{D}$ on $L^{2}\left(\mathbb{S}_{A}, \mu\right)$.

In particular, this means that one can apply to the Sierpinski set $\mathbb{S}_{A}$ all the techniques for constructions of wavelets on fractals from representations of Cuntz algebras developed, for instance, in [3,4,12-14,16-19,26-28], etc.

Notice then that we can embed the limit set $\Lambda_{A}$ inside the Sierpinski fractal $\mathbb{S}_{A}$ in the following way.

\section{Lemma 2.24 The map}

$$
\Xi: \Lambda_{A} \rightarrow \mathbb{S}_{A}, \quad \Xi(x)=(x, \sigma(x))
$$

gives an embedding $\Lambda_{A} \hookrightarrow \mathbb{S}_{A}$.

Proof A point $x=\left(x_{1} x_{2} \cdots x_{n} \cdots\right)$ in $\Lambda_{A}$ satisfies $A_{x_{i} x_{i+1}}=1$. This means that the point

$$
(x, y)=\left(0 . x_{1} x_{2} \cdots x_{n} \cdots, 0 . x_{2} x_{3} \cdots x_{n+1} \cdots\right)=(x, \sigma(x))
$$

satisfies $A_{x_{i}, y_{i}}=A_{x_{i} x_{i+1}}=1$ for all $i \geq 1$, hence it is a point in $\mathbb{S}_{A}$. The map $\Xi$ is clearly injective since it is the identity on the first coordinate. It is continuous since the preimage of a clopen set $\mathbb{S}_{A}\left(i_{1} \cdots i_{k}, j_{i} \cdots j_{k}\right)$ of $\mathbb{S}_{A}$, given by numbers with fixed first $k$ digits of the $N$-adic expansion, is either empty, or else, when $j_{r}=i_{r+1}$ for $r=1, \ldots, k-1$, it is equal to the clopen set $\Lambda_{A}\left(i_{1}, \ldots, i_{k}, j_{k}\right)$ of $\Lambda_{A}$.

One can then use this embedding together with the representation of the algebra $O_{D}$ on $L^{2}\left(\mathbb{S}_{A}, \mu\right)$ to obtain an induced action of a Cuntz-Krieger algebra.

Proposition 2.25 The maps $\tau_{i, j}$ restricts to maps defined on domains $D_{i, j} \subset \Xi\left(\Lambda_{A}\right)$. These determine a semibranching function system on $\Xi\left(\Lambda_{A}\right)$ which gives rise to a representation of the algebra $O_{\tilde{A}}$, where the $D \times D$-matrix $\tilde{A}$ is given by

$$
\tilde{A}_{(i, j),(\ell, k)}=\delta_{j, \ell} A_{j k} .
$$

Proof The condition that $\tau_{(i, j)}(x, \sigma(x))=\left(\tau_{i}(x), \tau_{j}(\sigma(x))\right.$ is in $\Xi\left(\Lambda_{A}\right)$ determines the domain $D_{(i, j)} \subset \Xi\left(\Lambda_{A}\right)$ to be

$$
D_{(i, j)}=\left\{(x, \sigma(x)) \in \Xi\left(\Lambda_{A}\right) \mid \sigma_{j} \sigma(x)=\sigma \sigma_{i}(x)\right\}=\Xi\left(R_{j}\right) .
$$


In fact, the condition that $A_{i j}=1$ implies that $R_{j} \subset D_{i}$ in $\Lambda_{A}$, so that $\Xi\left(D_{i} \cap R_{j}\right)=$ $\Xi\left(R_{j}\right)$. We identify the restriction of continuous functions on $\mathbb{S}_{A}$ to $\Xi\left(\Lambda_{A}\right)$ with continuous functions on $\Lambda_{A}$ and we write equivalently, with a slight abuse of notation, $f(x, \sigma(x))$ or $f(x)$. One then sees that

$$
f\left(\tau_{(i, j)}(x, \sigma(x))\right)=f\left(\sigma_{i}(x)\right) \chi_{R_{j}}(x)
$$

This induces an isometry on the Hilbert space $L^{2}\left(\Xi\left(\Lambda_{A}\right), \mu_{S}\right)$, where $\mu_{s}$ is the Hausdorff measure of dimension $s=\operatorname{dim}_{H}\left(\Xi\left(\Lambda_{A}\right)\right)$,

$$
\hat{S}_{(i, j)}^{*} f(x)=N^{s} \chi_{R_{i j}}(x) f(\sigma(x))
$$

since for a function $f(x, \sigma(x))$ on $\Xi\left(\Lambda_{A}\right)$ we have

$$
\chi_{R_{(i, j)}}(x, \sigma(x)) f\left(\sigma(x), \sigma^{2}(x)\right)=\chi_{R_{i j}}(x) f(\sigma(x)) .
$$

This has adjoint

$$
\hat{S}_{(i, j)}^{*} f(x)=N^{-s} \chi_{R_{j}}(x) f\left(\sigma_{i}(x)\right)
$$

We then obtain

$$
\hat{S}_{(i, j)} \hat{S}_{(i, j)}^{*} f(x)=\chi_{R_{i j}}(x) \chi_{R_{j}}(\sigma(x)) f\left(\sigma_{i} \sigma(x)\right)=\chi_{R_{i j}}(x) f(x)
$$

so that we have the relation

$$
\sum_{(i, j)} \hat{S}_{(i, j)} \hat{S}_{(i, j)}^{*}=1
$$

We also have

$$
\hat{S}_{(i, j)}^{*} \hat{S}_{(i, j)} f(x)=\chi_{R_{j}}(x) \chi_{R_{i j}}\left(\sigma_{i}(x)\right) f\left(\sigma \sigma_{i}(x)\right)=\chi_{R_{j}}(x) f(x) .
$$

Using the fact that

$$
\chi_{R_{j}}=\sum_{k} A_{j k} \chi_{R_{j k}}
$$

we then obtain the other relation in the form

$$
\hat{S}_{(i, j)}^{*} \hat{S}_{(i, j)}=\sum_{k} A_{j k} \hat{S}_{(j, k)} \hat{S}_{(j, k)}^{*}
$$

These correspond to the Cuntz-Krieger relations for the matrix $\tilde{A}$ of (2.71). 


\section{Wavelets on Fractals}

A general construction of wavelets on self-similar fractals was described in [15], see also [2]. The cases considered there correspond, from the point of view of semibranching function systems, to the case where the $\sigma_{i}$ are defined on all of $X$, as in the case of the Cuntz algebra. To adapt these constructions of wavelets to the main case we are interested in, which is the Cantor sets $\Lambda_{A}$ introduced above, one can use the representation of the Cuntz-Krieger algebra $O_{A}$ on $L^{2}\left(\Lambda_{A}, d \mu_{A}\right)$ that we considered in the previous sections, and again the Perron-Frobenius theory for the non-negative matrix $A$.

We begin by recalling briefly how the construction of [15] works in the case of a semibranching function system on a measure space $(X, \mu)$ where the $N$ maps $\sigma_{i}$ are defined on all of $X$. In this case one considers the $(m+1)$-dimensional linear space $\mathfrak{P}^{m}$ of polynomials on $\mathbb{R}$ of degree $\leq m$, and one denotes by $\mathfrak{S}_{0}$ the linear subspace of $L^{2}(X, d \mu)$, generated by the restrictions $\left.P\right|_{\Lambda_{A}}$ of polynomials in $\mathfrak{P}^{m}$. Under the condition that $X$ preserves Markov's inequality (see Sect. 4 of [15]), one knows that one still has $\operatorname{dim} \mathfrak{S}_{0}=m+1$. One then considers the linear subspace $\mathfrak{S}_{1} \subset L^{2}(X, d \mu)$ of functions $f \in L^{2}(X, d \mu)$ that are $\mu$-almost everywhere on $R_{i}=\sigma_{i}(X)$ restrictions $\left.P\right|_{R_{i}}$ of some polynomial $P \in \mathfrak{P}^{m}$. Clearly $\mathfrak{S}_{0} \subset \mathfrak{S}_{1}$ and $\operatorname{dim} \mathfrak{S}_{1}=N \operatorname{dim} \mathfrak{S}_{0}=N(m+1)$, and let $\phi^{\ell}$, for $\ell=1, \ldots, m+1$ be an orthonormal basis for $\mathfrak{S}_{0}$. One then considers the orthogonal complement $\mathfrak{S}_{1} \ominus \mathfrak{S}_{0}$, with a fixed choice of an orthonormal basis $\psi^{\rho}$, for $\rho=1, \ldots,(N-1)(m+1)$. The functions $\phi^{r}$ and $\psi^{\rho}$ provide the mother wavelets. One then considers the family of linear subspaces $\mathfrak{S}_{k}$ of $L^{2}(X, d \mu)$, of functions whose restriction to each subset $\sigma_{i_{1}} \circ \cdots \circ \sigma_{i_{k}}(X)$, agrees $\mu$-almost everywhere with the restriction to the same set of a polynomial in $\mathfrak{P}^{m}$. These satisfy $\mathfrak{S}_{0} \subset \mathfrak{S}_{1} \subset \cdots \mathfrak{S}_{k} \subset \cdots L^{2}(X, d \mu)$. Moreover, any function in $L^{2}(X, d \mu)$ can be approximated by elements in

$$
\mathfrak{S}_{0} \oplus \bigoplus_{k \geq 0}\left(\mathfrak{S}_{k+1} \ominus \mathfrak{S}_{k}\right)
$$

since in fact the polynomials of degree zero already suffice, as they give combinations of characteristic functions of the sets $\sigma_{i_{1}} \circ \cdots \circ \sigma_{i_{k}}(X)$. The wavelets are then obtained in [15] as

$$
\psi_{a}^{\rho}=\mu\left(\sigma_{a}(X)\right)^{-1 / 2} \psi^{\rho} \circ \sigma_{a}^{-1}
$$

for $a=\left(i_{1}, \ldots, i_{k}\right)$ and $\sigma_{a}=\sigma_{i_{1}} \circ \cdots \circ \sigma_{i_{k}}$.

We show now how to adapt this construction to the case of the Cantor sets $\Lambda_{A}$. For simplicity, we describe in full only the case where one only considers locally constant functions, that is, where one starts with the 1-dimensional space $\mathfrak{P}^{0}$. This is the case that is closest to the classical construction based on the Haar wavelets [11].

On the space $\Lambda_{A} \subset[0,1]$, with the Hausdorff measure $\mu=\mu_{A}$, let $\mathfrak{S}_{k}$ denote the linear subspaces of $L^{2}\left(\Lambda_{A}, d \mu_{A}\right)$ obtained as above, starting from the 1-dimensional space $\mathfrak{P}^{0}$. Let

$$
\left\{f^{\ell, k}\right\}_{k=0, \ldots, N-1 ; \ell=1, \ldots, d_{k}}
$$


with

$$
d_{k}=\#\left\{j \mid A_{k j}=1\right\}
$$

be a family of locally constant functions on $\Lambda_{A}$ such that the support of $f^{\ell, k}$ is contained in $R_{k}$ and

$$
\int_{R_{k}} \overline{f^{\ell, k}} f^{\ell^{\prime}, k}=\delta_{\ell, \ell^{\prime}}
$$

We also require that

$$
\int_{R_{k}} f^{\ell, k}=0, \quad \forall \ell=1, \ldots, d_{k} .
$$

Lemma 3.1 A family of functions $f^{\ell, k}$ as in (3.2), satisfying (3.4) and (3.5), can be constructed using linear combinations of characteristic functions $\chi_{R_{k j}}$, where $R_{k j}=$ $\Lambda_{2, A}(k j)$. The resulting $f^{\ell, k}$ give an orthonormal basis of the space $\mathfrak{S}_{2} \ominus \mathfrak{S}_{1}$.

Proof To see that linear combinations of characteristic functions $\chi_{R_{k j}}$ suffice to construct the functions $f^{\ell, k}$, notice first that the $\chi_{R_{k j}}$ give an orthogonal basis for the space $\mathfrak{S}_{2}$, which is of dimension $\operatorname{dim} \mathfrak{S}_{2}=\sum_{k} d_{k}$. We then write the $f^{\ell, k}$ in the form

$$
f^{\ell, k}=\sum_{j} A_{k j} c_{j}^{\ell, k} \chi_{R_{k j}},
$$

where the conditions (3.4) and (3.5) translate into conditions on the coefficients of the form

$$
\sum_{j} A_{k j} \bar{c}_{j}^{\ell, k} c_{j}^{\ell^{\prime}, k} p_{k j}=\delta_{\ell, \ell^{\prime}}
$$

where we use the notation

$$
p_{k j}=\mu\left(R_{k j}\right)=N^{-2 \delta_{A}} p_{j}
$$

according to (2.46), where $p=\left(p_{0}, \ldots, p_{N-1}\right)$ is the Perron-Frobenius eigenvector $A p=r(A) p$ for the non-negative matrix $A$. Similarly, the condition (3.5) becomes

$$
\sum_{j} A_{k j} c_{j}^{\ell, k} p_{k j}=N^{-2 \delta_{A}} \sum_{j} A_{k j} c_{j}^{\ell, k} p_{j}=0,
$$

where we again use (3.8).

Let us introduce the following notation for convenience. Consider on $\mathbb{C}^{d_{k}} \subset \mathbb{C}^{N}$ the inner product 


$$
\langle v, w\rangle_{k}:=\sum_{j} A_{k j} \bar{v}_{j} w_{j} p_{j} .
$$

Let $\mathcal{V}_{k}$ denote the orthogonal complement, in the inner product (3.10) on $\mathbb{C}^{d_{k}}$ of the vector $u=(1,1, \ldots, 1)$, and let $\left\{c^{\ell, k}=\left(c_{i}^{\ell, k}\right)\right\}_{\ell=1, \ldots, d_{k}-1}$ be an orthonormal basis of $\mathcal{V}_{k}$, in the inner product (3.10), namely

$$
\left\langle c^{\ell, k}, u\right\rangle_{k}=0, \quad \text { and } \quad\left\langle c^{\ell, k} c^{\ell^{\prime}, k}\right\rangle_{k}=\delta_{\ell, \ell^{\prime}} .
$$

Then for $c^{\ell, k}$ as above, one sees that the functions (3.6) are an orthonormal family satisfying the conditions (3.4) and (3.5).

The space spanned by the $f^{\ell, k}$ is contained in $\mathfrak{S}_{2}$ by construction. The condition (3.5) ensures that the functions $f^{\ell, k}$ are orthogonal to all the $\chi_{R_{k}}$, hence they are in $\mathfrak{S}_{2} \ominus \mathfrak{S}_{1}$. They span a space of dimension $\sum_{k}\left(d_{k}-1\right)=\sum_{k} d_{k}-N=\operatorname{dim} \mathfrak{S}_{2} \ominus \mathfrak{S}_{1}$.

Theorem 3.2 Suppose given an orthonormal basis $\left\{f^{\ell, r}\right\}$ for $\mathfrak{S}_{2} \ominus \mathfrak{S}_{1}$, constructed as in Lemma 3.1 above. Then the functions of the form

$$
\psi_{a}^{\ell, r}=S_{a} f^{\ell, r}
$$

for $a=\left(a_{1}, \ldots, a_{k}\right) \in \mathcal{W}_{k, A}$, give an orthonormal basis for the space $\mathfrak{S}_{k+1} \ominus \mathfrak{S}_{k}$ hence, for varying $a \in \mathcal{W}_{k, A}$ and for all $k \geq 0$, they give an orthonormal basis of wavelets for $L^{2}\left(\Lambda_{A}, \mu\right)$.

Proof We have shown in Lemma 3.1 that the functions $f^{\ell, r}$, for $r=0, \ldots, N-1$ and $\ell=1, \ldots, d_{r}$, give an orthonormal basis of $\mathfrak{S}_{2} \ominus \mathfrak{S}_{1}$. We then check that the functions $S_{a} f^{\ell, r}$ give an orthonormal basis for $\mathfrak{S}_{k+1} \ominus \mathfrak{S}_{k}$. Since in the representation of $O_{A}$ on $L^{2}\left(\Lambda_{A}, d \mu_{A}\right)$ we have constant Radon-Nikodym derivatives $\Phi_{\sigma_{i}}=N^{-\delta_{A}}$, this gives

$$
S_{j} f=N^{\delta_{A} / 2} \chi_{R_{j}} f \circ \sigma,
$$

so that we then have

$$
S_{a} f^{\ell, r}=N^{\delta_{A} k / 2} \chi_{\Lambda_{k, A}(a)} f^{\ell, r} \circ \sigma^{k}
$$

For $a \in \mathcal{W}_{k, A}$, we have

$$
\begin{aligned}
\left\langle S_{a} f^{\ell, r}, S_{a^{\prime}} f^{\ell^{\prime}, r^{\prime}}\right\rangle & =N^{\delta_{A} k}\left\langle\chi_{R_{a}} f^{\ell, r} \circ \sigma^{k}, \chi_{R_{a^{\prime}}} f^{\ell^{\prime}, r^{\prime}} \circ \sigma^{k}\right\rangle \\
& =N^{\delta_{A} k} \delta_{a, a^{\prime}} \int_{R_{a}} \overline{\left(f^{\ell, r} \circ \sigma^{k}\right)}\left(f^{\ell^{\prime}, r^{\prime}} \circ \sigma^{k}\right) d \mu,
\end{aligned}
$$

where we write $R_{a}=\Lambda_{k, A}(a)$, for the range of $\sigma_{a}=\sigma_{a_{1}} \circ \cdots \circ \sigma_{a_{k}}$. Notice then that we have, for any function $f \in L^{2}\left(\Lambda_{A}, d \mu\right)$ and any $a \in \mathcal{W}_{k, A}$, 


$$
\begin{aligned}
\int_{R_{a}} f \circ \sigma^{k} d \mu & =\int_{D_{a_{k}}} f \frac{d \mu \circ \sigma_{a}}{d \mu} d \mu \\
& =N^{-\delta_{A} k} \int_{D_{a_{k}}} f d \mu=N^{-\delta_{A} k} \sum_{j} A_{a_{k} j} \int_{R_{j}} f d \mu .
\end{aligned}
$$

Applied to the above this gives

$$
\left\langle S_{a} f^{\ell, r}, S_{a^{\prime}} f^{\ell^{\prime}, r^{\prime}}\right\rangle=\delta_{a, a^{\prime}} \delta_{r, r^{\prime}} A_{a_{k} r} \int_{R_{r}} \overline{f^{\ell, r}} f^{\ell^{\prime}, r} d \mu=\delta_{a, a^{\prime}} \delta_{r, r^{\prime}} \delta_{\ell, \ell^{\prime}} .
$$

Thus the $S_{a} f^{\ell, k}$ form an orthonormal system.

The space spanned by these functions is contained in $\mathfrak{S}_{k+1}$ and a counting of dimensions shows that it has the dimension of $\mathfrak{S}_{k+1} \ominus \mathfrak{S}_{k}$. To see that the $S_{a} f^{\ell, k}$ are in fact orthogonal to the elements of $\mathfrak{S}_{k}$ it suffices to compute

$$
\begin{aligned}
\left\langle S_{a} f^{\ell, r}, \chi_{\Lambda_{k, A}(b)}\right\rangle & =\delta_{a, b} N^{\delta_{A} k} \int_{R_{a}} f^{\ell, r} \circ \sigma^{k} d \mu \\
& =\delta_{a, b} \sum_{j} A_{a_{k}, j} \int_{R_{j}} f^{\ell, r} d \mu=\delta_{a, b} A_{a_{k}, r} \int_{R_{r}} f^{\ell, r} d \mu=0,
\end{aligned}
$$

by (3.13) and (3.5). This shows that we obtained an orthonornal basis of $\mathfrak{S}_{k+1} \ominus \mathfrak{S}_{k}$, hence a wavelet system for $L^{2}\left(\Lambda_{A}, d \mu\right)$.

It is useful to remark how the main difference in this case, as opposed to the similar constructions given for instance in [15] that we mentioned above, is that here we need to start from an orthonormal basis of $\mathfrak{S}_{2} \ominus \mathfrak{S}_{1}$ instead of $\mathfrak{S}_{1} \ominus \mathfrak{S}_{0}$. This reflects the fact that our functions $\sigma_{i}$ are not everywhere defined and, while the choice of an orthonormal basis for $\mathfrak{S}_{1} \ominus \mathfrak{S}_{0}$ gives the needed information on the ranges $R_{i}$, in order to control both the ranges and the domains $D_{i}$ one needs to go one step further before starting the induction that constructs the wavelets, and consider $\mathfrak{S}_{2} \ominus \mathfrak{S}_{1}$. Thus, the wavelet decomposition of a function $f \in L^{2}\left(\Lambda_{A}, \mu\right)$ will be given by

$$
f=\sum_{k=0}^{N-1} \sum_{\ell=1}^{d_{k}-1} \alpha_{\ell, k} f^{\ell, k}+\sum_{j=0}^{\infty} \sum_{a \in \mathcal{W}_{j, A}} \sum_{(\ell, k)} \alpha_{\ell, k, a} S_{a} f^{\ell, k}
$$

The more general case where one starts the wavelet construction from the linear space of polynomials $\mathfrak{P}^{m}$ with $m \geq 1$ can be done along the same lines as Lemma 3.1 and Theorem 3.2. We describe in the next section a different approach to wavelets constructions based on the Ruelle transfer operator for the coding map $\sigma$. This is closer to the point of view developed in [12]. 


\section{Ruelle Transfer Operator}

A more general version of the Perron-Frobenius operator associated to the coding map $\sigma: \Lambda_{A} \rightarrow \Lambda_{A}$ is obtained by considering the Ruelle transfer operator. This depends on the choice of a potential function $W$, defined on $\Lambda_{A}$, and is defined as

$$
\mathcal{R}_{\sigma, W} f(x)=\sum_{y: \sigma(y)=x} W(y) f(y)
$$

Lemma 4.1 If the function $W$ is real valued, one can describe the operator $\mathcal{R}_{\sigma, W}$ as the adjoint of the operator

$$
T_{W} f(x)=N^{\delta_{A}} W(x) f(\sigma(x)) .
$$

Proof We have

$$
\begin{aligned}
\left\langle T_{W} f, h\right\rangle & =\int_{\Lambda_{A}} N^{\delta_{A}} W(x) \overline{f(\sigma(x))} h(x) d \mu(x) \\
& =\sum_{i} \int_{D_{i}} \overline{f(u)} W\left(\sigma_{i}(u)\right) h\left(\sigma_{i}(u)\right) d \mu(u),
\end{aligned}
$$

using the fact that the Radon-Nikodym derivative $d \mu \circ \sigma_{i} / d \mu=N^{-\delta_{A}}$. We then write the above as

$$
\sum_{i, j} A_{i j} \int_{R_{j}} \overline{f(u)} W\left(\sigma_{i}(u)\right) h\left(\sigma_{i}(u)\right) d \mu(u)
$$

We also have

$$
\sum_{i, j} A_{i j} \chi_{R_{j}}(x) W\left(\sigma_{i}(x)\right) h\left(\sigma_{i}(x)\right)=\sum_{i} A_{i x_{1}} W\left(\sigma_{i}(x)\right) h\left(\sigma_{i}(x)\right) .
$$

Since the set of preimages of the point $x$ under the coding map is given by

$$
\{y \mid \sigma(y)=x\}=\bigcup_{i: A_{i x_{1}}=1} R_{i}
$$

we see that the above is in fact

$$
\sum_{i} A_{i x_{1}} W\left(\sigma_{i}(x)\right) h\left(\sigma_{i}(x)\right)=\sum_{y: \sigma(y)=x} W(y) f(y) .
$$

This shows that $\left\langle T_{W} f, h\right\rangle=\left\langle f, \mathcal{R}_{\sigma, W}(h)\right\rangle$. 
We assume that the potential $W$ of the Ruelle transfer operator satisfies the Keane condition, namely that it has non-negative real values $W: \Lambda_{A} \rightarrow \mathbb{R}_{+}$, and satisfies

$$
\sum_{y: \sigma(y)=x} W(y)=1 .
$$

Equivalently, this means

$$
\sum_{i} A_{i x_{1}} W\left(\sigma_{i}(x)\right)=1
$$

\subsection{Random Processes}

In the same way as described in [12], we relate here harmonic functions for the Ruelle transfer operator, that is, functions satisfying $\mathcal{R}_{\sigma, W} h=h$ to random processes defined by transition probabilities for paths from a given point $x$ to the image under the $\sigma_{j}$ and their iterates.

Let $A^{t}$ be the transpose of the matrix $A$. Then we have $a^{t}=\left(a_{k}, \ldots, a_{1}\right) \in \mathcal{W}_{k, A}$ if and only if $a=\left(a_{1}, \ldots, a_{k}\right) \in \mathcal{W}_{k, A^{t}}$. We construct probability measures on the limit set $\Lambda_{A^{t}}$ that are related to fixed points of the Ruelle transfer operator for the coding $\sigma: \Lambda_{A} \rightarrow \Lambda_{A}$. In the following we denote by $R_{i}$ and $D_{i}$, as before, the ranges and domains of the maps $\sigma_{i}$ in $\Lambda_{A}$ and by $R_{i}^{t}$ and $D_{i}^{t}$ the corresponding sets in $\Lambda_{A^{t}}$.

For a given potential $W$ on $\Lambda_{A}$ satisfying the Keane condition (4.3), consider a function $x \mapsto P_{x}^{W}$, for $x \in D_{i} \subset \Lambda_{A}$, where $P_{x}^{W}: \mathcal{B}\left(\Lambda_{A^{t}} \cap R_{i}^{t}\right) \rightarrow \mathbb{R}_{+}$, is a non-negative function on the Borel subsets of $\Lambda_{A^{t}}$ defined by assigning to the $\Lambda_{k, A^{t}}(a)$ the values

$$
P_{x}^{W}\left(\Lambda_{k, A^{t}}(a)\right)=A_{a_{1} x_{1}} W\left(\sigma_{a_{1}}(x)\right) W\left(\sigma_{a_{2}} \sigma_{a_{1}}(x)\right) \cdots W\left(\sigma_{a_{k}} \cdots \sigma_{a_{1}}(x)\right),
$$

for $a^{t}=\left(a_{k}, \ldots, a_{1}\right) \in \mathcal{W}_{k, A}$ and for $x \in D_{a_{1}} \subset \Lambda_{A}$.

Lemma 4.2 The assignment (4.5), for $x \in D_{i} \subset \Lambda_{A}$, defines a measure on $R_{i}^{t} \subset \Lambda_{A^{t}}$.

Proof Similarly, to the case of $\Lambda_{A}$ seen in (2.43), to check that (4.5) defines a measure one has to check the compatibility condition

$$
P_{x}^{W}\left(\Lambda_{k, A^{t}}(a)\right)=\sum_{j} A_{a_{k} j}^{t} P_{x}^{W}\left(\Lambda_{k+1, A}(a j)\right),
$$

for all $x \in D_{a_{1}} \subset \Lambda_{A}$. We have

$$
\left.P_{x}^{W}\left(\Lambda_{k+1, A}(a j)\right)=A_{a_{1}, x_{1}} W\left(\sigma_{a_{1}}(x)\right) \cdots W\left(\sigma_{a_{k}} \cdots \sigma_{a_{1}}(x)\right) W\left(\sigma_{j} \sigma_{a_{k}} \cdots \sigma_{a_{1}}(x)\right)\right)
$$

Moreover, the Keane condition for $W$ on $\Lambda_{A}$ gives

$$
\left.\sum_{j} A_{j a_{k}} W\left(\sigma_{j} \sigma_{a_{k}} \cdots \sigma_{a_{1}}(x)\right)\right)=1
$$

so we obtain (4.6). 
One can think of the values of the potential $W$ as defining a probability of transition, or walk, from $x$ to $\sigma_{a_{1}}(x)$, so that (4.5) can be regarded as the probability of a random walk from $x$ to $\sigma_{a_{k}} \cdots \sigma_{a_{1}}(x)$. We then see that the random process $P_{x}^{W}$ is related to the fixed points of the Ruelle transfer operator.

Proposition 4.3 The random process $x \mapsto P_{x}^{W}$ introduced above is related to fixed points of the Ruelle transfer operator in the following ways.

(1) Let $E \subset \Lambda_{A^{t}}$ be a shift invariant set $\sigma^{-1}(E)=E$. Then the function $x \mapsto$ $P_{x}^{W}(E)$ is a fixed point of the Ruelle transfer operator with potential $W$ on $\Lambda_{A}$.

(2) If the series

$$
h(x):=\sum_{k \geq 1} \sum_{a \in \mathcal{W}_{k, A^{t}}} A_{a_{1} x_{1}} W\left(\sigma_{a_{1}}(x)\right) \cdots W\left(\sigma_{a_{k}} \cdots \sigma_{a_{1}}(x)\right)
$$

converges, then the function $h(x)$ is a fixed point of the Ruelle transfer operator with potential $W$ on $\Lambda_{A}$.

Proof (1) We check that this condition is equivalent to the fixed point condition under the Ruelle transfer operator. For a given set $\Lambda_{k, A^{t}}(a)$, we have

$$
\begin{aligned}
\mathcal{R}_{\sigma, W}\left(P_{x}^{W}\left(\Lambda_{k, A^{t}}(a)\right)\right) & =\sum_{y: \sigma(y)=x} W(y) P_{y}^{W}\left(\Lambda_{k, A^{t}}(a)\right) \\
& =\sum_{j} A_{j x_{1}} W\left(\sigma_{j}(x)\right) P_{\sigma_{j}(x)}^{W}\left(\Lambda_{k, A^{t}}(a)\right) .
\end{aligned}
$$

A shift invariant set $\sigma^{-1}(E)=E$ in $\Lambda_{A^{t}}$ satisfies

$$
\cup_{j, i: A_{j i}^{t}=1} \sigma_{j}\left(E \cap R_{i}\right)=E .
$$

By construction of the measures $P_{x}^{W}$, we know that $P_{x}^{W}\left(\sigma_{j}\left(E \cap R_{i}^{t}\right)\right)$ is non-trivial provides that $x \in D_{j}$, so that $A_{j x_{1}}=1$. Thus, for $\sigma^{-1}(E)=E$, we have

$$
\mathcal{R}_{\sigma, W}\left(P_{x}^{W}(E)\right)=\sum_{j} A_{i j} P_{x}^{W}\left(\sigma_{j}\left(E \cap R_{i}^{t}\right)\right)=P_{x}^{W}\left(\sigma^{-1}(E)\right)=P_{x}^{W}(E),
$$

which shows that $P_{x}^{W}(E)$ is a fixed point for $\mathcal{R}_{\sigma, W}$.

(2) Assuming that the series (4.7) converges, we have

$$
\begin{aligned}
\mathcal{R}_{\sigma, W} h(x) & =\sum_{\sigma(y)=x} W(y) h(y)=\sum_{j} A_{j x_{1}} W\left(\sigma_{j}(x)\right) h\left(\sigma_{j}(x)\right) \\
& =\sum_{j} A_{j x_{1}} W\left(\sigma_{j}(x)\right) \sum_{k} \sum_{a} A_{a_{1} j} W\left(\sigma_{a_{1}} \sigma_{j}(x)\right) \cdots W\left(\sigma_{a_{k}} \cdots \sigma_{a_{1}} \sigma_{j}(x)\right) \\
& =\sum_{k} \sum_{b=j a \in W_{k+1, A^{t}}} A_{j x_{1}} W\left(\sigma_{j}(x)\right) W\left(\sigma_{a_{1}} \sigma_{j}(x)\right) \cdots W\left(\sigma_{a_{k}} \cdots \sigma_{a_{1}} \sigma_{j}(x)\right) .
\end{aligned}
$$

This gives $\mathcal{R}_{\sigma, W} h(x)=h(x)$. 


\subsection{A Trigonometric Example}

We give an example of a potential $W$ satisfying the Keane condition, constructed using trigonometric functions.

Lemma 4.4 The function

$$
W(x)=\frac{1}{N_{1}}\left(1-\cos \left(\frac{2 \pi N x}{N_{1}}\right)\right),
$$

with $N_{1}=\#\left\{j: A_{j x_{1}}=1\right\}$, is a potential satisfying the Keane condition (4.3) on $\Lambda_{A}$.

Proof First notice that we have

$$
\sum_{j=0}^{N-1} A_{j x_{1}} \exp \left(\frac{2 \pi i N \sigma_{j}(x)}{N_{1}}\right)=0,
$$

since $\sigma_{j}(x)=(x+j) / N$ and the above becomes a sum over all the $N_{1}$ th roots of unity. It follows directly from this that the real valued trigonometric version also satisfies

$$
\sum_{j=0}^{N-1} A_{j x_{1}} \cos \left(\frac{2 \pi N \sigma_{j}(x)}{N_{1}}\right)=0
$$

from which it follows that the potential of (4.8) satisfies

$$
\sum_{j=0}^{N-1} A_{j x_{1}} W\left(\sigma_{j}(x)\right)=1 .
$$

Moreover, the function $W(x)$ takes non-negative real values, so it gives a potential with the Keane condition.

\section{Examples and Applications}

5.1 Hensley Cantor Sets and Continued Fraction Expansion

In [23] the coding of geodesics on the modular curves $X_{\Gamma}=\mathbb{H} / \Gamma$, for $\Gamma \subset \mathrm{PGL}_{2}(\mathbb{Z})$ a finite index subgroup and $\mathbb{H}$ the hyperbolic upper half plane, was related to a generalization of the shift map of the continued fraction expansion $T:[0,1] \times \mathbb{P} \rightarrow[0,1] \times \mathbb{P}$,

$$
T(x, s)=\left(\frac{1}{x}-\left[\frac{1}{x}\right],\left(\begin{array}{cc}
-[1 / x] & 1 \\
1 & 0
\end{array}\right) s\right),
$$

where $\mathbb{P}=\mathrm{PGL}_{2}(\mathbb{Z}) / \Gamma$ is the finite coset set. It was then shown in [24,25], that the restriction of this dynamical system to the Hensley Cantor sets, that is, those subsets 
$E_{N} \subset[0,1]$ of points that only contains digits $a_{k} \leq N$ in the continued fraction expansion, gives rise to a dynamical system

$$
\sigma: E_{N} \times \mathbb{P} \rightarrow E_{N} \times \mathbb{P}
$$

which can be identified with the coding map $\sigma: \Lambda_{A} \rightarrow \Lambda_{A}$ of a semibranching function system $\left\{\sigma_{i}\right\}$ that determines a Cuntz-Krieger algebra $O_{A}$. The case where $\Gamma=\mathrm{PGL}_{2}(\mathbb{Z})$ recovers the Cuntz algebra $O_{N}$.

In this setting, one considers the Ruelle transfer operator with potential (without Keane condition)

$$
W(x, s)=\left|T^{\prime}(x, s)\right|^{\beta}
$$

so that

$$
\begin{aligned}
\mathcal{R}_{T, W} f(x, s) & =\sum_{T(y, t)=(x, s)}\left|T^{\prime}(y, t)\right|^{\beta} f(y, t) \\
& =\sum_{n=1}^{N} \frac{1}{(x+n)^{2 \beta}} f\left(\frac{1}{x+n},\left(\begin{array}{ll}
0 & 1 \\
1 & n
\end{array}\right) s\right) .
\end{aligned}
$$

This can be written in the form

$$
\sum_{(n, t)} A_{(n, t),\left(x_{1}, s\right)} W\left(\sigma_{(n, t)}(x, s)\right) f\left(\sigma_{(n, t)}(x, s)\right),
$$

where the matrix $A$ is defined by the condition

$$
A_{(n, t),(k, s)}= \begin{cases}1 & M_{n} s=t \\ 0 & \text { otherwise }\end{cases}
$$

where the matrix $M_{n} \in \mathrm{GL}_{2}(\mathbb{Z})$, acting on the left on the coset $\mathbb{P}$, is

$$
M_{n}=\left(\begin{array}{ll}
0 & 1 \\
1 & n
\end{array}\right)
$$

The shift invariant measure $\mu_{N, \mathbb{P}}$ on $E_{N} \times \mathbb{P}$ constructed in [24] using the fixed point of the Ruelle transfer operator can then be also seen as in [22] as $\mathrm{KMS}_{\beta}$ state for the time evolution on the Cuntz-Krieger algebra $O_{A}$ given by

$$
\sigma_{t}\left(S_{(k, s)}\right)=W^{-i t} S_{(k, s)},
$$

where we identify $W^{-i t}$, for fixed $t$, with an element in $C\left(E_{N} \times \mathbb{P}\right)$. The KMS state is then of the form

$$
\varphi_{\beta}\left(S_{a} S_{a}^{*}\right)=\int_{E_{N} \times \mathbb{P}} f_{a}(x, s) d \mu_{N, \mathbb{P}}(x, s),
$$


for $a=\left(\left(k_{1}, s_{1}\right), \ldots,\left(k_{r}, s_{r}\right)\right) \in \mathcal{W}_{r, A}$ and $f_{a}$ the element in $C\left(E_{N} \times \mathbb{P}\right)$ that corresponds to $S_{a} S_{a}^{*}$. The Ruelle operator can correspondingly be written as

$$
\mathcal{R}_{T, W} f=\sum_{(n, t)} S_{(n, t)}^{*} W f S_{(n, t)}
$$

in term of generators of the Cuntz-Krieger algebra.

\subsection{Graph Wavelets from Cuntz-Krieger Algebras}

It was recently shown, see for instance [8], that the crucial problem of spatial traffic analysis on networks can be addressed using a form of wavelet analysis which is adapted to the topology of the network graph. These graph wavelets are constructed as families of functions $\Psi_{\alpha}(v)$ on the set of vertices $V(G)$ of a given finite graph $G$, localized with respect to certain scaling indices $\alpha$, and with the property that

$$
\int_{V(G)} \Psi_{\alpha}(v) d \mu(v)=0, \quad \text { and } \int_{V(G)} \bar{\Psi}_{\alpha}(v) \Psi_{\alpha^{\prime}}(v) d \mu(v)=\delta_{\alpha, \alpha^{\prime}},
$$

where $\mu(v)$ is a given measure that weights the nodes of the network with assigned probabilities. We show here how to construct families of graph wavelets using the representations of Cuntz-Krieger algebras and the corresponding wavelets on $\Lambda_{A}$ constructed in Sect. 3.

Let $G$ be a finite directed graph with no sinks. It is well known that one can associate to such a graph a Cuntz-Krieger algebra in the following way. One considers a collection of projections $P_{v}$ associated to the vertices $v \in V(G)$ and a collection of partial isometries $S_{e}$ associated to the oriented edges $e \in E(G)$, with the relations

$$
P_{v}=\sum_{s(e)=v} S_{e} S_{e}^{*}
$$

for all $v \in V(G)$, and

$$
P_{r(e)}=S_{e}^{*} S_{e},
$$

for all edges $e \in E(G)$. Assuming that the graph has no sinks, so that all vertices are sources, one has $\sum_{v} P_{v}=1$ so that the isometries $S_{e}$ satisfy the relation (2.2),

$$
\sum_{e} S_{e} S_{e}^{*}=1
$$

Moreover, for $N=\# E(G)$, one defines the $N \times N$-matrix $A_{e e^{\prime}}$ by

$$
A_{e e^{\prime}}= \begin{cases}1 & r(e)=s\left(e^{\prime}\right) \\ 0 & \text { otherwise }\end{cases}
$$


Then the relation (5.5) reads equivalently as

$$
S_{e}^{*} S_{e}=\sum_{e^{\prime}: r(e)=s\left(e^{\prime}\right)} S_{e^{\prime}} S_{e^{\prime}}^{*}=\sum_{e^{\prime}} A_{e e^{\prime}} S_{e^{\prime}} S_{e^{\prime}}^{*},
$$

which gives the other Cuntz-Krieger relation (2.1).

As before, let $\Lambda_{A}$ be the limit set associated to the algebra $O_{A}$ of the graph $G$. Let $d_{e}=\#\left\{e^{\prime} \mid r(e)=s\left(e^{\prime}\right)\right\}=\#\left\{e^{\prime} \mid A_{e e^{\prime}}=1\right\}$. Consider as in Sect. 3 the orthonormal family of functions $\left\{f^{\ell, e}\right\}$ with $e \in E(G)$ and $\ell=1, \ldots, d_{e}$. As we have seen in Sect. 3 these are the mother wavelets for the orthonormal basis of $L^{2}\left(\Lambda_{A}, \mu_{A}\right)$ given by the functions $\left\{S_{a} f^{\ell, e}\right\}$, for varying $a \in \mathcal{W}_{k, A}$ and $k \in \mathbb{N}$. Here an element $a=\left(e_{1}, \ldots, e_{k}\right) \in \mathcal{W}_{k, A}$ is a path in the graph $G$ of length $k$ starting at the vertex $s\left(e_{1}\right)$. Here we use the same mother functions to construct a family of graph wavelets.

Recall from Sect. 3 that the functions $f^{\ell, e}$ are constructed in terms of a family $c^{\ell, e}=\left(c_{e^{\prime}}^{\ell, e}\right)$ of vectors satisfying

$$
\sum_{e^{\prime}} A_{e, e^{\prime}} \bar{c}_{e^{\prime}}^{\ell, e} c_{e^{\prime}}^{\ell^{\prime}, e} p_{e e^{\prime}}=\delta_{e, e^{\prime}}
$$

where $p_{e e^{\prime}}=\mu\left(R_{e e^{\prime}}\right)=N^{-2 \delta_{A}} p_{e^{\prime}}$ and

$$
\sum_{e^{\prime}} A_{e, e^{\prime}} c_{e^{\prime}}^{\ell, e} p_{e^{\prime}}=0
$$

Upon rescaling the coefficients $c_{e^{\prime}}^{\ell, e}$ by a factor $N^{\delta_{A}}$, we obtain a family satisfying (5.10) and with (5.9) replaced by the similar

$$
\sum_{e^{\prime}} A_{e, e^{\prime}} \bar{c}_{e^{\prime}}^{\ell, e} c_{e^{\prime}, e}^{\ell^{\prime}, e} p_{e^{\prime}}=\delta_{e, e^{\prime}}
$$

where we keep the same notation for these rescaled coefficients. The $p_{e}$ are the components of the Perron-Frobenius eigenvector $A p=r(A) p$.

After fixing a choice of a base vertex $v_{0} \in E(G)$, we define a measure on the set of vertices of the graph by $\mu_{G, v_{0}}\left(v_{0}\right)=0$ and

$$
\mu_{G, v_{0}}(v):=p_{e_{1}} \cdots p_{e_{k}}
$$

where $e_{1} \cdots e_{k}$ is the shortest path in the graph $G$ starting at $v_{0}$ and ending at $v$. This means that we are considering a random walk on the graph starting at $v_{0}$, where at the first step one has probability $p_{e}$ of moving to the nearby vertex $r(e)$ and probability zero of remaining at $v_{0}$. The measure (5.12) gives the probability of reaching at time $k$ one of the vertices that are $k$ steps away from $v_{0}$. 
In addition to fixing the base vertex $v_{0}$, we also fix a choice of an edge $e_{0}$ with $r\left(e_{0}\right)=v_{0}$. We then define functions

$$
\Psi_{\ell}(v)= \begin{cases}c_{e^{\prime}}^{\ell, e_{0}} & v=r\left(e^{\prime}\right), v_{0}=r\left(e_{0}\right)=s\left(e^{\prime}\right) \\ 0 & \text { otherwise. }\end{cases}
$$

These satisfy

$$
\int_{V(G)} \Psi_{\ell}(v) d \mu_{G, v_{0}}(v)=\sum_{e^{\prime}} A_{e_{0} e^{\prime}} c_{e^{\prime}}^{\ell, e_{0}} p_{e^{\prime}}=0
$$

and

$$
\int_{V(G)} \bar{\Psi}_{\ell}(v) \Psi_{\ell^{\prime}}(v) d \mu_{G, v_{0}}(v)=\sum_{e^{\prime}} A_{e_{0} e^{\prime}} \bar{c}_{e^{\prime}}^{\ell, e_{0}} c_{e^{\prime}}^{\ell^{\prime}, e_{0}} p_{e^{\prime}}=\delta_{\ell, \ell^{\prime}}
$$

We then extend this to a family $\Psi_{\ell_{1}, \ldots, \ell_{k}}(v)$, where we consider paths $a=\left(e_{1}, \ldots, e_{k}\right) \in$ $\mathcal{W}_{k, A}$ of length $k$ in the graph starting at $v_{0}$, with $\ell_{i}=1, \ldots, d_{e_{i}}$. We set

$$
\Psi_{\ell_{1}, \ldots, \ell_{k}}(v)= \begin{cases}c_{e_{1}}^{\ell_{1}, e_{0}} c_{e_{2}}^{\ell_{2}, e_{1}} \cdots c_{e_{k}}^{\ell_{k}, e_{k-1}} & v=r\left(e_{k}\right), v_{0}=s\left(e_{1}\right), \\ 0 & \text { otherwise. }\end{cases}
$$

These again satisfy

$$
\begin{aligned}
& \int_{V(G)} \Psi_{\ell_{1}, \ldots, \ell_{k}}(v) d \mu_{G, v_{0}}(v) \\
& \quad=\sum_{\left(e_{1}, \ldots, e_{k}\right)} A_{e_{0} e_{1}} \cdots A_{e_{k-1} e_{k}} c_{e_{1}}^{\ell_{1}, e_{0}} c_{e_{2}}^{\ell_{2}, e_{1}} \cdots c_{e_{k}}^{\ell_{k}, e_{k-1}} p_{e_{1}} \cdots p_{e_{k}}=0 .
\end{aligned}
$$

This vanishes since already $\sum_{e_{k}} A_{e_{k-1} e_{k}} c_{e_{k}}^{\ell_{k}, e_{k-1}} p_{e_{k}}=0$. Moreover, they satisfy

$$
\begin{aligned}
& \int_{V(G)} \bar{\Psi}_{\ell_{1}, \ldots, \ell_{k}}(v) \Psi_{\ell_{1}^{\prime}, \ldots, \ell_{k}^{\prime}}(v) d \mu_{G, v_{0}}(v) \\
& \quad=\sum_{\left(e_{1}, \ldots, e_{k}\right)} A_{e_{0} e_{1}} \cdots A_{e_{k-1} e_{k}} \bar{c}_{e_{1}}^{\ell_{1}, e_{0}} \cdots \bar{c}_{e_{k}}^{\ell_{k}, e_{k-1}} c_{e_{1}}^{\ell_{1}^{\prime}, e_{0}} \cdots c_{e_{k}}^{\ell_{k}^{\prime}, e_{k-1}} p_{e_{1}} \cdots p_{e_{k}} \\
& =\delta_{\ell_{1}, \ell_{1}^{\prime}} \cdots \delta_{\ell_{k}, \ell_{k}^{\prime}}
\end{aligned}
$$

The functions $\Psi_{\ell_{1}, \ldots, \ell_{k}}$, for $k \geq 1$, constructed in this way, are supported on concentric regions $\mathcal{U}_{k}\left(v_{0}\right)$ made of vertices at a distance $k$ from a chosen base vertex $v_{0}$. Unlike other types of graph wavelets constructions where the functions are constant on such concentric regions $\mathcal{U}_{k}\left(v_{0}\right)$ and average to zero over different $k$, the ones we obtain here are supported on a single $\mathcal{U}_{k}\left(v_{0}\right)$ with zero average. In terms of traffic analysis on 
networks, while one type of graph wavelets may be more suitable in analyzing radial propagation from a vertex, the other may be preferable for directional propagation away from a chosen vertex.

In $[6,7]$ one considered, in the setting of Mumford curves with p-adic Schottky uniformization, the Cuntz-Krieger algebras associated to the finite graphs with no sinks obtained from the action of a p-adic Schottky group on the subtree of the Bruhat-Tits tree spanned by geodesics with boundary points on the limit set in $\mathbb{P}^{1}\left(\mathbb{Q}_{p}\right)$. In that context it would be interesting to compare the wavelet constructions described in this paper with the p-adic wavelet theory (see for instance [1]).

Acknowledgments Part of this work was done during a stay of the authors at the Max Planck Institute for Mathematics, which we thank for the hospitality and support. M. Marcolli was partially supported by NSF grant DMS-0651925.

Open Access This article is distributed under the terms of the Creative Commons Attribution Noncommercial License which permits any noncommercial use, distribution, and reproduction in any medium, provided the original author(s) and source are credited.

\section{References}

1. Albeverio, S., Kozyrev, S.V.: Multidimensional basis of p-adic wavelets and representation theory. arXiv:0903.0461

2. Bodin, M.: Wavelets and Besov spaces on Mauldin-Williams fractals. Real Anal. Exch. 32(1), 119144 (2006)

3. Bratteli, O., Jorgensen, P.E.T.: Iterated function systems and permutation representations of the Cuntz algebra. Mem. Am. Math. Soc. 139, 663 (1999)

4. Bratteli, O., Jorgensen, P.E.T., Ostrowsky, V.: Representation theory and numerical AF-invariants. The representations and centralizers of certain states on $O_{d}$. Mem. Am. Math. Soc. 168, xviii+178pp (2004)

5. Consani, C., Marcolli, M.: Noncommutative geometry, dynamics, and $\infty$-adic Arakelov geometry. Selecta Math. (N.S.) 10(2), 167-251 (2004)

6. Consani, C., Marcolli, M.: Spectral triples from Mumford curves. Int. Math. Res. Not. 36, 1945-1972 (2003)

7. Cornelissen, G., Marcolli, M., Reihani, K., Vdovina, A.: Noncommutative geometry on trees and buildings. In: Traces in Number Theory, Geometry and Quantum Fields. Aspects of Mathematics, vol. E38, pp. 73-98. Vieweg, Braunschweig (2008)

8. Crovella, M., Kolaczyk, E.: Graph wavelets for spatial traffic analysis. In: Proceedings of IEEE Infocom 2003, San Francisco, CA, USA, April (2003)

9. Cuntz, J., Krieger, W.: A class of $C^{*}$-algebras and topological Markov chains. Invent. Math. 56(3), 251268 (1980)

10. Davidson, K.R., Pitts, D.R.: Invariant subspaces and hyper-reflexivity for free semigroups algebras. Proc. Lond. Math. Soc. 78, 401-430 (1999)

11. Daubechies, I.: Ten lectures on wavelets. In: CBMS-NSF Regional Conference Series in Applied Mathematics, vol. 61. Society for Industrial and Applied Mathematics, Philadelphia (1992)

12. Dutkay, D.E., Jorgensen, P.E.T.: Iterated function systems, Ruelle operators, and invariant projective measures. Math. Comp. 75, 1931-1970 (2006)

13. Dutkay, D.E., Jorgensen, P.E.T.: Methods from multiscale theory and wavelets applied to nonlinear dynamics. In: Wavelets, Multiscale Systems and Hypercomplex Analysis. Operator Theory: Advances and Applications vol. 167, pp. 87-126. Birkhäuser, Basel (2006)

14. Dutkay, D.E., Jorgensen, P.E.T.: Wavelets on fractals. Rev. Mat. Iberoam. 22(1), 131-180 (2006)

15. Jonsson, A.: Wavelets on fractals and Besov spaces. J. Fourier Anal. Appl. 4(3), 329-340 (1998)

16. Jorgensen, P.E.T.: Iterated function systems, representations, and Hilbert space. Int. J. Math. 15(8), 813832 (2004) 
17. Jorgensen, P.E.T.: Use of operator algebras in the analysis of measures from wavelets and iterated function systems. In: Operator Theory, Operator Algebras, and Applications. Contemporary Mathematics, vol. 414, pp. 13-26. American Mathematical Society, Providence (2006)

18. Jorgensen, P.E.T.: Measures in wavelet decompositions. Adv. Appl. Math. 34(3), 561-590 (2005)

19. Jorgensen, P.E.T., Song, M.S.: Optimal decompositions of translations of $L^{2}$-functions. Complex Anal. Oper. Theory 2(3), 449-478 (2008)

20. Kawamura, K.: The Perron-Frobenius operators, invariant measures and representations of the Cuntz-Krieger algebra. J. Math. Phys. 46, 083514 (2005)

21. Kawamura, K.: Permutative representations of the Cuntz-Krieger algebras. arXiv:math/0508273

22. Kesseböhmer, M., Stadlbauer, M., Stratmann, B.O.: Lyapunov spectra for KMS states on Cuntz-Krieger algebras. Math. Z. 256, 871-893 (2007)

23. Manin, Yu.I., Marcolli, M.: Continued fractions, modular symbols, and noncommutative geometry. Selecta Math. (New Ser.) 8(3), 475-521 (2002)

24. Marcolli, M.: Limiting modular symbols and the Lyapunov spectrum. J. Number Theory 98(2), 348-376 (2003)

25. Marcolli, M.: Modular curves, $C^{*}$-algebras, and chaotic cosmology. In: Frontiers in Number Theory, Physics and Geometry, II, pp. 361-372. Springer, Berlin (2007)

26. Strichartz, R.S.: Wavelet expansions of fractal measures. J. Geom. Anal. 1(3), 269-289 (1991)

27. Strichartz, R.S.: Wavelets and self-affine tilings. Constr. Approx. 9(2-3), 327-346 (1993)

28. Strichartz, R.S.: Piecewise linear wavelets on Sierpinski gasket type fractals. J. Fourier Anal. Appl. 3(4), 387-416 (1997) 